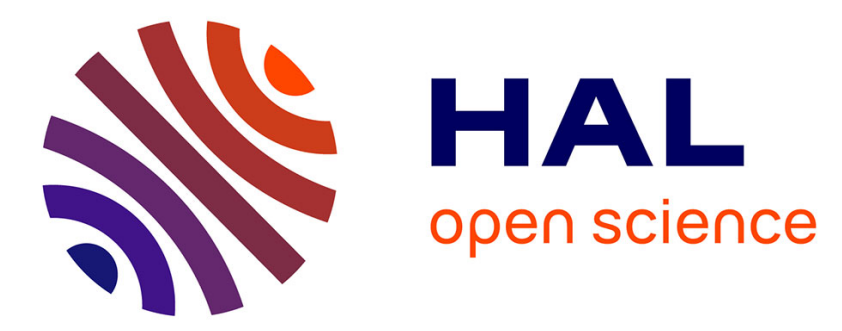

\title{
Multi-objective Hydrodynamic Optimization of the DTMB 5415 for Resistance and Seakeeping
}

Matteo Diez, Andrea Serani, Emilio F. Campana, O. Goren, K. Sarioz, D.B.

Danisman, G. Grigoropoulos, E. Aloniat, Michel Visonneau, Patrick Queutey, et al.

\section{To cite this version:}

Matteo Diez, Andrea Serani, Emilio F. Campana, O. Goren, K. Sarioz, et al.. Multi-objective Hydrodynamic Optimization of the DTMB 5415 for Resistance and Seakeeping. 13th International Conference on Fast Sea Transportation - FAST 2015, Sep 2015, Washington DC, United States. hal-01202600

\section{HAL Id: hal-01202600 \\ https://hal.science/hal-01202600}

Submitted on 8 Oct 2020

HAL is a multi-disciplinary open access archive for the deposit and dissemination of scientific research documents, whether they are published or not. The documents may come from teaching and research institutions in France or abroad, or from public or private research centers.
L'archive ouverte pluridisciplinaire HAL, est destinée au dépôt et à la diffusion de documents scientifiques de niveau recherche, publiés ou non, émanant des établissements d'enseignement et de recherche français ou étrangers, des laboratoires publics ou privés. 


\title{
Multi-objective hydrodynamic optimization of the DTMB 5415 for resistance and seakeeping
}

\author{
Matteo Diez ${ }^{1,2}$, Andrea Serani ${ }^{1,3}$, Emilio F. Campana ${ }^{1}$, Omer Goren ${ }^{4}$, Kadir Sarioz ${ }^{4}$, \\ D. Bulent Danisman ${ }^{4}$, Gregory Grigoropoulos ${ }^{5}$, Eleni Aloniati ${ }^{5}$, Michel Visonneau ${ }^{6}$, \\ Patrick Queutey ${ }^{6}$, Frederick Stern ${ }^{2}$
}

\author{
${ }^{1}$ CNR-INSEAN, National Research Council-Marine Technology Research Institute, Rome, Italy \\ ${ }^{2}$ The University of Iowa, IIHR-Hydroscience and Engineering, Iowa City, IA, USA \\ ${ }^{3}$ Roma Tre University, Rome, Italy \\ ${ }^{4}$ ITU, Istanbul Technical University, Istanbul, Turkey \\ ${ }^{5}$ NTUA, National Technical University of Athens, Athens, Greece \\ ${ }^{6} \mathrm{ECN}$, Ecole Centrale de Nantes, CNRS, Nantes, France
}

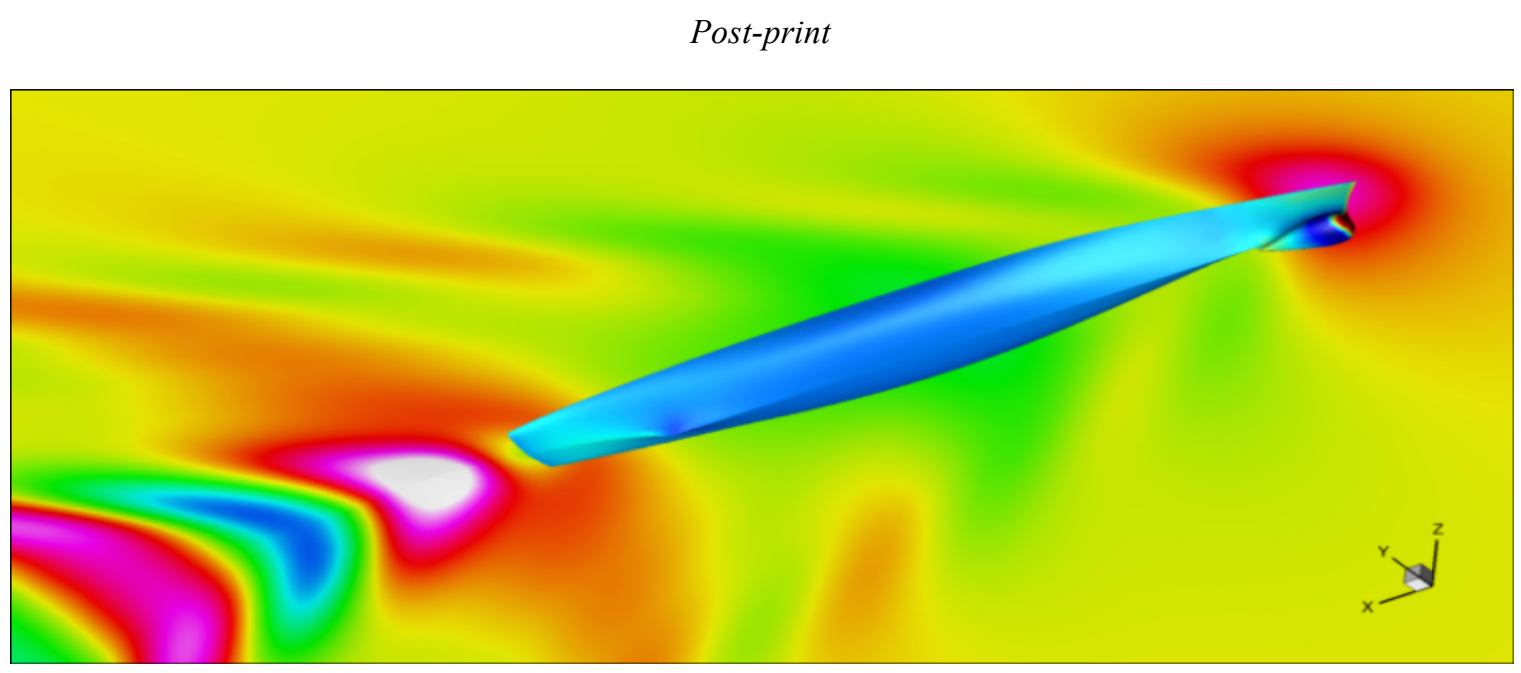

Abstract: The paper presents recent research conducted within the NATO RTO Task Group AVT-204 "Assess the Ability to Optimize Hull Forms of Sea Vehicles for Best Performance in a Sea Environment." The objective is the improvement of the hydrodynamic performances (resistance/powering requirements, seakeeping, etc.) of naval vessels, by integration of computational methods used to generate, evaluate, and optimize hull-form variants. Several optimization approaches are brought together and compared. A multiobjective optimization of the DTMB 5415 (specifically the MARIN variant 5415M) is used as a test case and results obtained so far using low-fidelity solvers show an average improvement for resistance and seakeeping performances of nearly 10 and $9 \%$, respectively.

Keywords: Simulation-based design optimization; Hydrodynamic optimization; Hull-form optimization; Multi-objective optimization; Ship design; DTMB 5415.

\section{INTRODUCTION}

In order to reduce costs and improve the performance for a variety of missions, navies are demanding new concepts and multicriteria optimized ships. In order to address this challenge, research teams have developed simulation-based design optimization (SBDO) methods, to generate hull variants and optimize their hydrodynamic performance, combining low- and high-fidelity solvers, design modification tools, and multiobjective optimization algorithms. The NATO RTO Task Group AVT-204, formed to "Assess the Ability to Optimize Hull Forms of Sea Vehicles for Best Performance in a Sea Environment," addresses the integration and assessment of different computational methods and SBDO approaches, bringing together teams from France (ECN, Ecole Centrale de Nantes/CNRS),
Germany (TUHH, Hamburg University of Technology), Greece (NTUA, National Technical University of Athens), Italy (INSEAN, National Research Council-Marine Technology Research Institute), Turkey (ITU, Istanbul Technical University), and Unites States (UI, University of Iowa).

The objective is the development of a greater understanding of the potential and limitations of the hydrodynamic optimization tools and their integration within SBDO. The former include lowand high-fidelity solvers, automatic shape modification tools, and multi-objective optimization algorithms, and are limited in the present activity to deterministic applications.

The approach includes SBDO methods from different research teams, which are assessed and compared. At the current stage of 
the activities, INSEAN and UI are undertaking a joint effort for a two phase SBDO, using low-fidelity solvers in the first phase, and more accurate and computationally expensive high-fidelity solvers in the second phase. ITU and NTUA have performed separate SBDO procedures, based on low-fidelity solvers, whereas ECN is using high-fidelity solvers to verify low-fidelity optimization outcomes. ECN and TUHH will address respectively maneuvering and propulsion performances, as part of future activities.

SBDO tools and results are presented in the following, for each research team separately. Analysis tools used in the current study include potential flow (INSEAN/UI, ITU, NTUA) and RANSE $(\mathrm{ECN})$ solvers. Design modification tools include linear expansion of orthogonal basis functions (INSEAN/UI), an approach based on relaxation coefficients at control points with Akima's surface generation (ITU), and the parametric modelling of the CAESES/FRIENDSHIP-Framework, which parametrizes the hull by 19 sections, using a set of basic curves, with associated topological information (NTUA). Multi-objective optimization algorithms include a multi-objective extension of the deterministic particle swarm optimization algorithm (INSEAN/UI), a sequential quadratic programming method, which is applied to an artificial neural network model of aggregate objective functions (ITU), and a non-dominated sorting genetic algorithm (NTUA).

The test case of the current study is the deterministic hull-form optimization of a USS Arleigh Burke-class destroyer, namely the DDG-51. The DTMB 5415 model, an open-to-public early concept of the DDG-51, is used for the current research. This has been largely investigated through towing tank experiments (e.g., Stern et al., 2000; Longo and Stern, 2005), and used for earlier SBDO research for conventional (Tahara et al., 2008) and hybrid Kandasamy et al., 2014) hulls. Both 5415 bare hull (INSEAN/UI, ECN) and the $5415 \mathrm{M}$ variant with skeg only (ITU, NTUA) are addressed. The design optimization exercise aims at the reduction of two objective functions, namely (i) the weighted sum of the total resistance in calm water at 18 and $30 \mathrm{kn}$ (corresponding to $\mathrm{Fr}=0.25$ and 0.41 ), and (ii) a seakeeping merit factor based on the vertical acceleration of the bridge (in head wave, sea state 5, $\mathrm{Fr}=0.41$ ) and the roll motion (in stern wave, sea state $5, \mathrm{Fr}=0.25$ ). The first speed for resistance optimization $(18 \mathrm{kn})$ is close to the peak of the speed-time profile for transits, from 2013 data (Anderson et al., 2013). The second speed (20 kn) is the flank speed, used as an objective to minimize the maximum powering requirements. The seakeeping merit factor is based on a first extreme condition, and on a second, less extreme, condition. Sea state 5 is considered as an average open ocean condition for North Atlantic and North Pacific, year round (Bales, 1983; Lee, 1995).

\section{DTMB 5415 MULTI-OBJECTIVE OPTIMIZATION}

The full-scale main particulars are summarized in Table 1. The optimization aims at improving both calm-water and seakeeping performances, and is formulated as

$$
\begin{array}{cl}
\text { Minimize } & F 1(\mathbf{x}), F 2(\mathbf{x}) \\
\text { Subject to } G_{k}(\mathbf{x})=0, & k=1, \ldots, E \\
\text { and to } H_{k}(\mathbf{x}) \leq 0, & k=1, \ldots, I
\end{array}
$$

where $\mathbf{x}$ is the design variable vector, $F 1$ is the weighted sum of the normalized total resistance in calm water at $18(\mathrm{Fr}=0.25)$ and $30 \mathrm{kn}(\mathrm{Fr}=0.41)$, respectively,

$$
F 1(\mathbf{x})=\left.0.85 \frac{R_{T}}{R_{T_{0}}}\right|^{18 k t}+\left.0.15 \frac{R_{T}}{R_{T_{0}}}\right|^{30 k t}
$$

with $R_{T_{o}}$ the total resistance of the parent hull, and $F 2$ is a seakeeping merit factor, defined as

$$
F 2(\mathbf{x})=\left.0.5 \frac{R M S\left(a_{z}\right)}{R M S\left(a_{z_{0}}\right)}\right|_{180^{\circ}} ^{30 k n}+\left.0.5 \frac{R M S(\varphi)}{R M S\left(\varphi_{0}\right)}\right|_{30^{\circ}} ^{18 k n}
$$

where $R M S$ represents the root mean square, $a_{z}$ is the vertical acceleration of the bridge (located $27 \mathrm{~m}$ forward amidships and $24.75 \mathrm{~m}$ above keel) at $30 \mathrm{kn}$ in head wave, and $\varphi$ is the roll angle at $18 \mathrm{kn}$ in stern long-crested wave. The wave conditions correspond to sea state 5 , using the Bretschneider spectrum with a significant wave height of $3.25 \mathrm{~m}$ and a modal period of $9.7 \mathrm{~s}$.

Geometrical equality constraints $\left(G_{k}\right)$ include fixed length between perpendiculars and displacement, whereas geometrical inequality constraints $\left(H_{k}\right)$ include limited variation of beam and draught $( \pm 5 \%)$ and reserved volume for the sonar in the dome, corresponding to $4.9 \mathrm{~m}$ diameter and $1.7 \mathrm{~m}$ length (cylinder).

Table 1. DTMB 5415 main particulars (full scale)

\begin{tabular}{|l|c|c|c|}
\hline Description & Symbol & Unit & Value \\
\hline Displacement & $\mathrm{D}$ & ton & 8,636 \\
\hline $\begin{array}{l}\text { Length between } \\
\text { perpendiculars }\end{array}$ & $\mathrm{LBP}$ & $\mathrm{m}$ & 142 \\
\hline Beam & $\mathrm{B}$ & $\mathrm{m}$ & 18.9 \\
\hline $\begin{array}{l}\text { Longitudinal center of } \\
\text { gravity }\end{array}$ & $\mathrm{LCG}$ & $\mathrm{m}$ & 71.6 \\
\hline Vertical center of gravity & $\mathrm{VCG}$ & $\mathrm{m}$ & 1.39 \\
\hline Roll radius of gyration & $\mathrm{K}_{\mathrm{xx}}$ & - & $0.40 \mathrm{~B}$ \\
\hline Pitch radius of gyration & $\mathrm{K}_{\mathrm{yy}}$ & - & $0.25 \mathrm{LBP}$ \\
\hline Yaw radius of gyration & $\mathrm{K}_{\mathrm{zz}}$ & - & $0.25 \mathrm{LBP}$ \\
\hline
\end{tabular}

\section{INSEAN/UI}

The SBDO framework, used for the first optimization phase by INSEAN/UI, integrates low-fidelity solvers for calm-water resistance and seakeeping prediction, a design modification method based on linear expansion of orthogonal basis function, and a multi-objective optimization algorithm based on the particle swarm metaheuristic, which are described in the following. The tool box is applied to the DTMB 5415 bare hull.

In the second optimization phase, the SBDO will be performed substituting the low fidelity solvers with RANSE, using a sequential multi-criterion adaptive sampling technique with a dynamic radial basis function model (Diez et al., 2015).

\section{INSEAN/UI - Low-fidelity Solvers}

$\boldsymbol{W A R P}$. The WAve Resistance Program is a linear potential flow code, in-house developed at INSEAN. The Neumann-Kelvin 
linearization is used for the current optimization study. Details of equations, numerical implementations and validation of the numerical solver are given in Bassanini et al. (1994).

For optimization purposes, the wave resistance is evaluated by the transverse wave cut method (Telste and Reed, 1994), whereas the frictional resistance is estimated using a flat-plate approximation, based on the local Reynolds number (Schlichting and Gersten, 2000). The steady 2 DOF (sinkage and trim) equilibrium is achieved by iteration of the flow solver and the body equation of motion.

SMP. The Standard Ship Motion program was developed at the David Taylor Naval Ship Research and Development Center in 1981, as a prediction tool for use in the Navy's ship design process. SMP provides a potential flow solution based on linearized strip theory. The 6 DOF response of the ship is given, advancing at constant forward speed with arbitrary heading in both regular waves and irregular seas, as well as the longitudinal, lateral, and vertical responses at specified locations of the ship (Meyers and Baitis, 1981).

\section{INSEAN/UI - Design Modification Method}

Shape modifications $\boldsymbol{\delta}_{S}$ are produced by superposition of orthogonal basis functions $\boldsymbol{\Psi}_{j}$, and controlled by $N_{d v}$ design variables $\alpha_{j}$, as

$$
\boldsymbol{\delta}_{S}(\xi, \eta)=\sum_{j=1}^{N_{d v}} \alpha_{j} \boldsymbol{\Psi}_{j}(\xi, \eta)
$$

with

$$
\boldsymbol{\Psi}_{j}(\xi, \eta):=\sin \left(\frac{p_{j} \pi \xi}{A_{j}-B_{j}}+\phi_{j}\right) \sin \left(\frac{q_{j} \pi \eta}{C_{j}-D_{j}}+\chi_{j}\right) \mathbf{e}_{k(j)}
$$

where $(\xi, \eta) \in\left[A_{j} ; B_{j}\right] \times\left[C_{j} ; D_{j}\right]$ are curvilinear coordinates; $p_{j}$ and $q_{j}$ define the order of the function in $\xi$ and $\eta$ direction respectively; $\phi_{j}$ and $\chi_{j}$ are the corresponding spatial phases; $A_{j}, B_{j}, C_{j}$ and $D_{j}$ define the patch size; $\mathbf{e}_{k(j)}$ is a unit vector. Modifications may be applied in $x, y$, or $z$ direction, by setting $k(j)=1,2$ or 3 , respectively (Serani et al., 2015b).

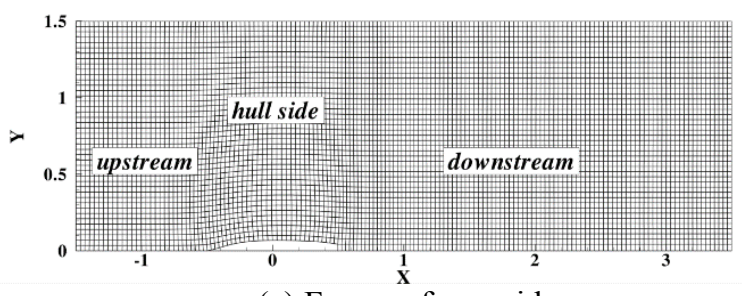

(a) Free-surface grid
Once the shape modification is produced over the selected surface-body patches using Eq. 4, geometrical equality constraints are satisfied by automatic scaling.

\section{INSEAN/UI - Optimization Algorithm}

A multi-objective extension of the deterministic particle swarm optimization algorithm (MODPSO) is used for the present study. The advantage of using a deterministic version of the algorithm is that a statistical analysis of the results is not necessary (see, e.g., Chen et al., 2015). The MODPSO iteration is given by

$$
\left\{\begin{array}{l}
\mathbf{v}_{i}^{n+1}=\chi\left[\mathbf{v}_{i}^{n}+c_{1}\left(\mathbf{x}_{i, p b}-\mathbf{x}_{i}^{n}\right)+c_{2}\left(\mathbf{x}_{i, g b}-\mathbf{x}_{i}^{n}\right)\right] \\
\mathbf{x}_{i}^{n+1}=\mathbf{x}_{i}^{n}+\mathbf{v}_{i}^{n+1}
\end{array}\right.
$$

for $i=1, \ldots, N_{p}$, where $N_{p}$ represents the swarm size (number of particles); $\mathbf{x}_{i}^{n}$ is the position of the $i$-th particle at the $n$-th iteration; $\mathbf{x}_{i, p b}$ and $\mathbf{x}_{i, g b}$ are the personal (cognitive) and global (social) best positions associated to the $i$-th particle. Specifically, $\mathbf{x}_{i, p b}$ is the closest point to $\mathbf{x}_{i}^{n}$ of the personal (cognitive) Pareto front, whereas $\mathbf{x}_{i, g b}$ is the closest point to $\mathbf{x}_{i}^{n}$ of the global (social) Pareto front (see, e.g., Diez et al., 2010). The coefficients $\chi, c_{1}$ and $c_{2}$ in Eq. 6 control the swarm dynamics and affect the convergence of the algorithm.

The setup suggested by Pellegrini et al. (2014) is used for the current optimization. Specifically, $N_{p}$ is set equal to 16 times the number of design variables. The initialization of the particle swarm is based on a Hammersley sequence sampling (Wong et al., 1997) over variable domain and bounds, with non-null velocity (Chen et al., 2015). The set of coefficients is taken from Trelea (2003), setting $\chi=0.6, c_{1}=c_{2}=1.7$. A semi-elastic walltype approach is used for box constraints (Serani et al., 2014).

During swarm optimization, geometrical equality constraints are automatically satisfied by the shape modification tool, whereas inequality constraints are treated by a constant penalty function.

\section{INSEAN/UI - Numerical Results}

Numerical results include grid studies and comparison to EFD of the potential flow solvers, the design space definition with the sensitivity analysis of the design variables, and finally a summary

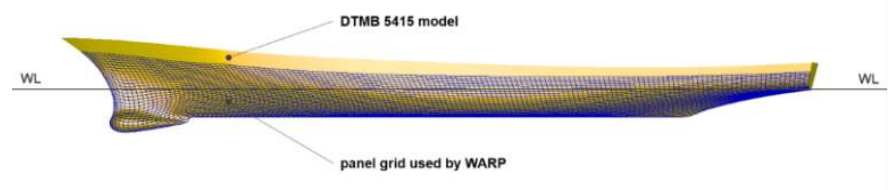

(b) Body grid

Figure 1. INSEAN - Computational panel grid (G1)

Table 2. INSEAN/UI - Potential flow solver solution for the original hull

\begin{tabular}{|c|c|c|c|c|c|}
\hline Fr & $C_{T} \times 10^{-3}$ & $\delta(\mathrm{m})$ & $\tau(\mathrm{deg})$ & $\begin{array}{c}R M S\left(a_{z}\right)\left(\mathrm{m} / \mathrm{s}^{2}\right), \\
\text { head wave }\end{array}$ & $\begin{array}{c}R M S(\phi)(\mathrm{deg}), \\
30 \text { deg stern wave }\end{array}$ \\
\hline 0.25 & 2.643 & 0.134 & 0.052 & - & 0.477 \\
\hline 0.41 & 6.068 & 0.424 & -0.412 & 0.902 & - \\
\hline
\end{tabular}




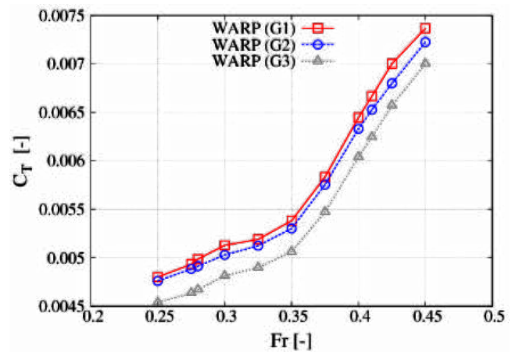

(a) Total resistance coefficient

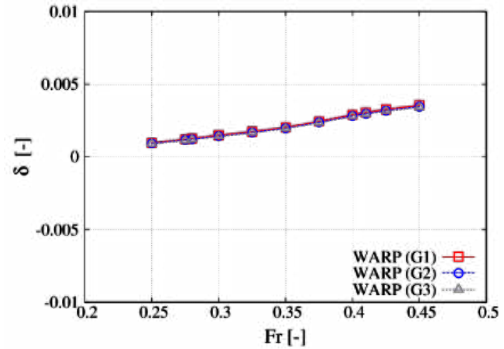

(b) Non-dimensional sinkage

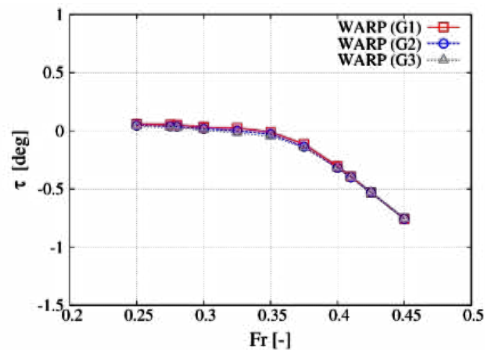

(c) Trim

Figure 2. INSEAN/UI - Panel-grid convergence analysis (WARP)

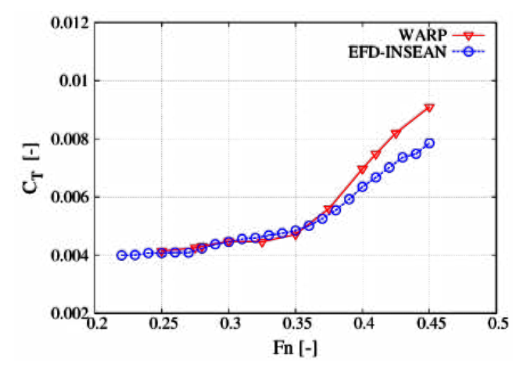

(a) Total resistance coefficient (WARP)

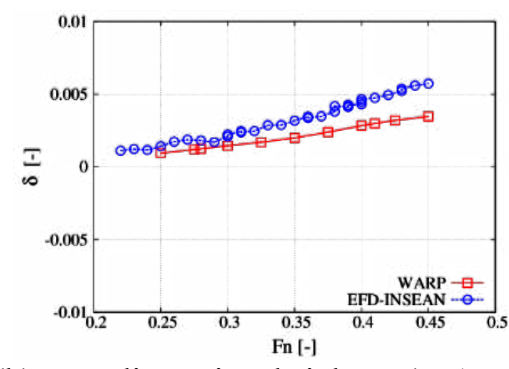

(b) Non-dimensional sinkage (WARP)

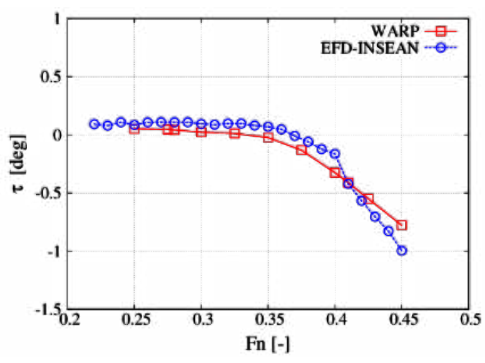

(c) Trim (WARP)

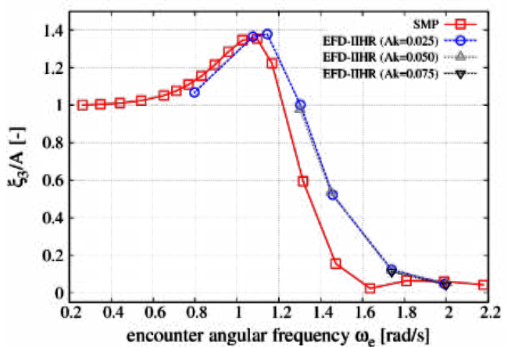

(d) Heave RAO in head wave, Fr=0.41 (SMP)

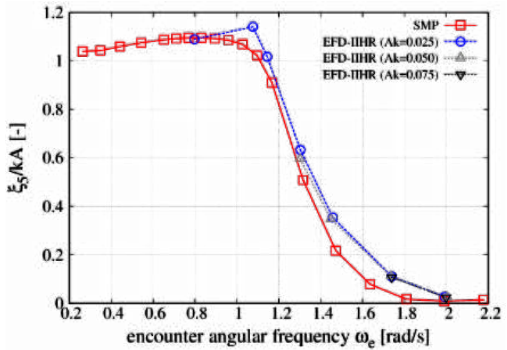

(e) Pitch RAO in head wave, Fr=0.41 (SMP)
Figure 3. INSEAN/UI - Potential flow results compared to EFD

of the design optimization results obtained using six different research spaces. Detailed results may be found in Serani et al. (2015a).

\section{Grid Studies and Comparison with EFD of the Potential Flow}

Solvers. The computational domain (WARP) for the free-surface is defined within one hull length upstream, three lengths downstream and 1.5 lengths for the side (Fig. 1a). One panel grid triplet $(\mathrm{G} 1, \mathrm{G} 2, \mathrm{G} 3)$ is used, with a refinement ratio equal to $\sqrt{2}$, and size equal to $11 \mathrm{k}, 5.5 \mathrm{k}$ and $2.8 \mathrm{k}$, respectively. Figure $1 \mathrm{~b}$ shows the body grid (G1) for the DTMB 5415 under consideration. The fluid condition are: $\rho=998.5 \mathrm{~kg} / \mathrm{m}^{3}, v=$ $1.09 \mathrm{E}-06 \mathrm{~m}^{2} / \mathrm{s}$ and $g=9.8033 \mathrm{~m} / \mathrm{s}^{2}$.

Calm-water potential flow results for the three panel grids are given in Fig. 2, with $\mathrm{Fr}$ varying from 0.25 to $0.45 ; C_{T}=$ $R_{T} /\left(0.5 \rho \mathrm{Fr}^{2} g \mathrm{LBP} S_{w, s t a t}\right), \delta$, and $\tau$ are shown, where $R_{T}$ is the total resistance, $S_{w, s t a t}$ is the static wetted surface area, $\delta$ is the sinkage (positive if the center of gravity sinks), and $\tau$ is the trim (positive if the bow sinks). The calm-water total resistance coefficient is found monotonic grid convergent, whereas sinkage and trim are monotonic divergent. Nevertheless, solution changes are very small and grid G1 is deemed adequate for current studies. Figures $3 \mathrm{a}, 3 \mathrm{~b}$, and $3 \mathrm{c}$ show a comparison with EFD data from INSEAN (Olivieri et al, 2001), revealing a reasonable agreement especially for low speeds.

Seakeeping results are presented in Figs. 3d and 3e in terms of heave and pitch response amplitude operators (RAOs) at $\mathrm{Fr}=0.41$ and head wave. The trend is compared to UI data (Longo and Stern, 2005), showing a reasonable agreement at least for peak values and peak frequencies. Table 2 summarizes the main results for the original hull.

Design Space Definition and Sensitivity Analysis. Four orthogonal basis functions and associated design variables are used for the hull, whereas two functions/variables are used for the sonar dome, as summarized in Table 3. Three design spaces are assessed:

A. Two-dimensional design space defined by the first and the third patches of Table 3, which are characterized by a first order function over the whole hull. The shape modification consists in moving volume back/front $(j=1)$ and down/up $(j=3)$. 
B. Four-dimensional design space defined by the two patches of A, along with two additional patches controlling the design of the sonar dome, reducing/increasing its width $(j=5)$ and moving the whole dome up/down $(j=6)$.

C. Six-dimensional design space defined by the four patches of $\mathrm{B}$, along with two additional patches $(j=2,4)$, which introduce a higher-order representation of the hull modifications (see Table 3).

For each design space, the optimization is performed using two domains, defined by the following box constraints:

Domain $1 . \quad-0.5 \leq x_{k} \leq 0.5, k=1, \ldots, 6$

Domain 2. $-1.0 \leq x_{k} \leq 1.0, k=1, \ldots, 6$ where

$$
x_{j}=2\left(\alpha_{j}-\alpha_{j, \min }\right) / 2\left(\alpha_{j, \max }-\alpha_{j, \min }\right)-1
$$

Domain 2 is an extensions of domain 1, with enlarged design variability.

A preliminary sensitivity analysis is performed, for calm water at $\mathrm{Fr}=0.25(18 \mathrm{kn})$ and $\mathrm{Fr}=0.41(30 \mathrm{kn})$, and for the seakeeping merit factor. The objective functions, $F 1$ and $F 2$, are shown in Fig. 4. Unfeasible designs are not reported. The results show a potential reduction close to $12 \%$ and $4 \%$ for $F 1$ and $F 2$, respectively.

Design Optimization and Selection of the Optimized Hull. The maximum number of function evaluations is set equal to 256 times the number of design variables. The selection of the optimal hull from the Pareto front is based on the best compromise between the two objective functions. Specifically, the selected solution has minimum $\Delta F 1+\Delta F 2$. Table 4 shows the nondimensional design variables of the selected optimal hull for each design space, and the objective function reduction. Table 5 summarized the optimization results for $F 1$, in terms of forces and dynamic sinkage and trim. Table 6 summarizes the optimization results for $F 2$, in terms of $R M S$ of vertical acceleration of the bridge and roll motion.

The design space C. 1 has produced the most promising design, with an improvement of nearly $6.7 \%$ and $6.8 \%$ for $F 1$ and $F 2$, respectively. It may be noted that the design spaces C.2 and B.2 provide an average reduction of the objective functions $F 1$ and F2 larger than C.1 (Table 4). Nevertheless, C.2 design also gives

Table 3. INSEAN/UI - Orthogonal functions parameters, for shape modification

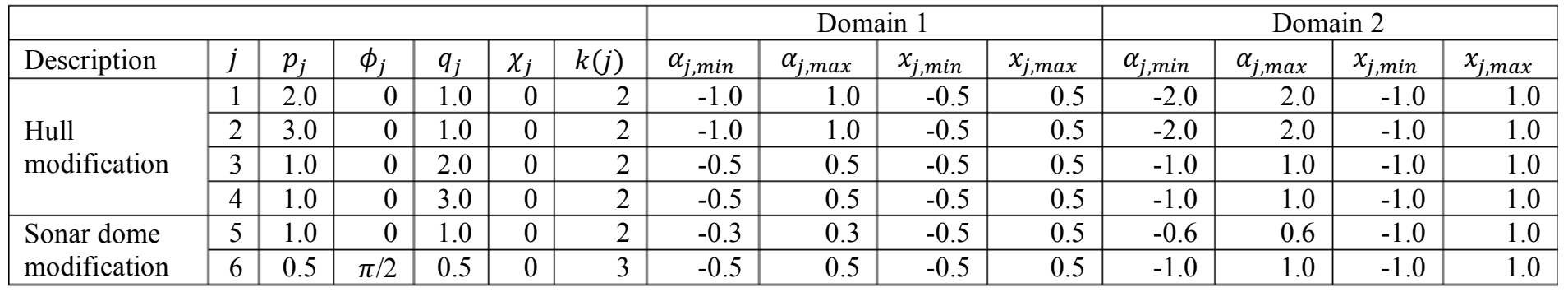

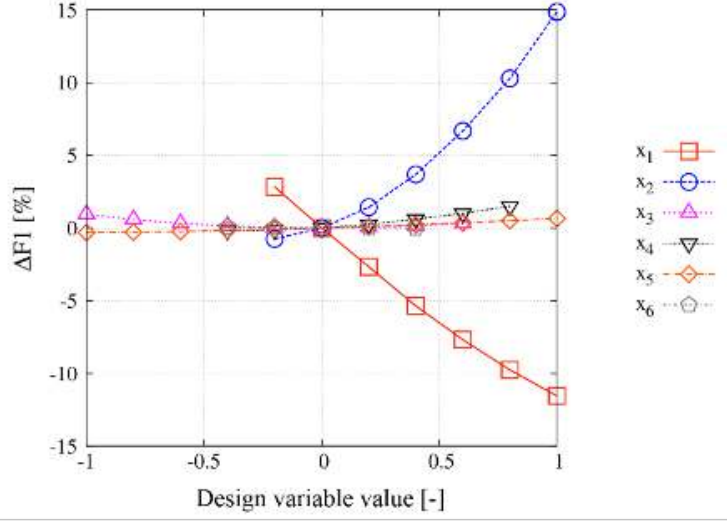

(a) $F 1$

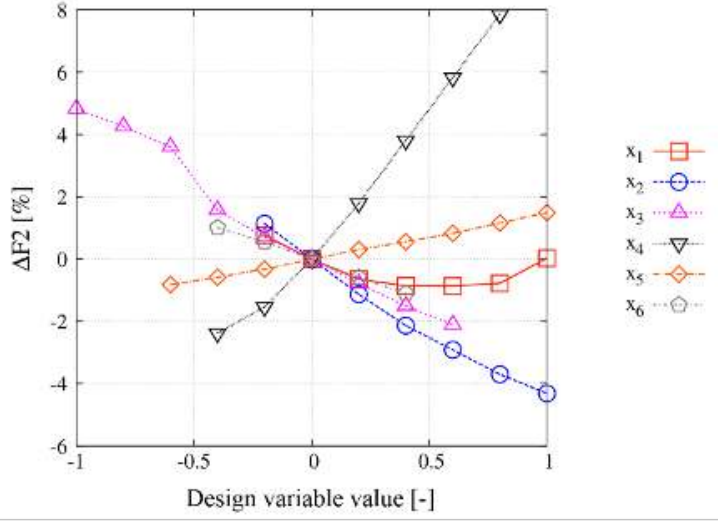

(b) $F 2$

Figure 4. INSEAN/UI - Sensitivity analysis of the design variables

Table 4. INSEAN/UI - Multi-objective deterministic particle swarm optimization results

\begin{tabular}{|c|c|c|c|c|c|c|c|c|c|}
\hline & \multicolumn{6}{|c|}{ Design variables (non-dimensional) } & \multicolumn{3}{|c|}{ Objective functions } \\
\hline DoE & $x_{1}$ & $x_{2}$ & $x_{3}$ & $x_{4}$ & $x_{5}$ & $x_{6}$ & $\Delta F 1 \%$ & $\Delta F 2 \%$ & Average $\%$ \\
\hline A.1 & 0.499 & & 0.500 & & & & -6.4 & -2.2 & -4.3 \\
\hline A. 2 & 1.000 & & 0.737 & & & & -11.4 & -1.6 & -7.5 \\
\hline B.1 & 0.500 & & 0.457 & & -0.493 & 0.413 & -6.6 & -4.1 & -5.3 \\
\hline B. 2 & 0.994 & & 0.361 & & -1.000 & 0.406 & -11.7 & -4.1 & -8.0 \\
\hline C. 1 & 0.496 & -0.021 & 0.327 & -0.479 & -0.489 & 0.431 & -6.7 & -6.8 & -6.8 \\
\hline C. 2 & 0.959 & -0.268 & -0.983 & -0.489 & -0.901 & 0.580 & -10.4 & -9.1 & -9.8 \\
\hline
\end{tabular}


Table 5. INSEAN/UI - Summary results for selected designs $(F 1)$

\begin{tabular}{|c|c|c|c|c|c|c|c|c|c|c|c|c|c|c|c|c|c|c|c|c|c|}
\hline \multirow[b]{2}{*}{$\mathrm{Fr}$} & \multirow[b]{2}{*}{ DoE } & \multicolumn{2}{|c|}{$C_{w} \times 10^{-3}$} & \multicolumn{2}{|c|}{$C_{f} \times 10^{-3}$} & \multicolumn{2}{|c|}{$C_{T} \times 10^{-3}$} & \multicolumn{2}{|c|}{$R_{w} \times 10^{6}(\mathrm{~N})$} & \multicolumn{2}{|c|}{$R_{f} \times 10^{5}(\mathrm{~N})$} & \multicolumn{2}{|c|}{$R_{T} \times 10^{6}(\mathrm{~N})$} & \multicolumn{2}{|c|}{$\mathrm{S}_{\mathrm{w}, \mathrm{stat}}\left(\mathrm{m}^{2}\right)$} & \multicolumn{2}{|c|}{$\mathrm{S}_{\mathrm{w}, \mathrm{dyn}}\left(\mathrm{m}^{2}\right)$} & \multicolumn{2}{|c|}{$\delta(\mathrm{m})$} & \multicolumn{2}{|c|}{$\tau(\mathrm{deg})$} \\
\hline & & ralue & $\Delta \%$ & ralue & $\Delta \%$ & alue & $\Delta \%$ & value & $\Delta \%$ & value & $\Delta \%$ & value & $\Delta \%$ & value & $\Delta \%$ & ralue & $\Delta \%$ & lue & $\Delta \%$ & lue & $\%$ \\
\hline \multirow{6}{*}{0.2} & 4 & 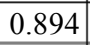 & & 598 & 0 & 402 & & & & . & 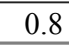 & & & 017 & & 050 & & & & & 05 \\
\hline & A.2 & & 6.7 & 8 & 0.0 & 63 & 10.6 & & & & & & & & & & & & & & \\
\hline & $\mathrm{B}$ & & 4.2 & 597 & .1 & 493 & & & & & 0 & & 0.0 & & & 0 & 1 & & & & \\
\hline & B.2 & & & 596 & .1 & & & & & & & & & & & & & & & & \\
\hline & & & & & & & & & & & & & & & & & & & & & \\
\hline & 0 & & & 598 & 0.1 & $\theta$ & & & & & 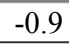 & & 10 & & & 07 & & & & & 4.2 \\
\hline \multirow{6}{*}{0.4} & $\mathrm{~A}$ & & .9 & 553 & -0.5 & 117 & 5.7 & & -2 & 75 & 0 & 4 & 9 & 7 & & 48 & 51 & 85 & .2 & & 1.6 \\
\hline & A & & & & - & & & & & & & & & & & & & & & & \\
\hline & \begin{tabular}{|l|} 
B.1 \\
\end{tabular} & & & .552 & -0.6 & 204 & & & -18.6 & 46 & -0 & & & & & & & 384 & -9.5 & & 38.2 \\
\hline & B.2 & 82 & 1.6 & .543 & -1.1 & 4.626 & - & & -31.6 & 96 & -1.1 & & .7 & & & 14 & -0.48 & 0.360 & -15.1 & & 90.9 \\
\hline & \begin{tabular}{|l|} 
C.1 \\
\end{tabular} & & & 1.551 & -0.6 & 5.237 & & 290 & -18.1 & .426 & -0 & & & 2995 & & 3123 & & 0.376 & -11.2 & & 41.4 \\
\hline & C. 2 & 810 & -15.5 & 1.553 & -0.5 & 5.362 & -11.6 & 1.320 & -16.2 & 5.378 & -1.4 & 1.857 & -12.4 & 2965 & -0.88 & 3093 & -1.15 & 0.386 & -8.8 & 0.083 & -120. \\
\hline
\end{tabular}

Table 6. INSEAN/UI - Summary results for selected designs (F2)

\begin{tabular}{|c|c|c|c|c|}
\hline \multirow[b]{2}{*}{ DoE } & \multicolumn{2}{|c|}{$\begin{array}{c}\mathrm{Fr}=0.41 \\
\text { head wave }(180 \mathrm{deg})\end{array}$} & \multicolumn{2}{|c|}{$\begin{array}{c}\mathrm{Fr}=0.25 \\
\text { stern wave }(30 \mathrm{deg})\end{array}$} \\
\hline & $\operatorname{RMS}\left(a_{z}\right)\left(\mathrm{m} / \mathrm{s}^{2}\right)$ & $\Delta \%$ & $R M S(\phi)(\mathrm{deg})$ & $\Delta \%$ \\
\hline A.1 & 0.825 & -8.6 & 0.497 & 4.2 \\
\hline A. 2 & 0.775 & -14.1 & 0.527 & 10.9 \\
\hline B.1 & 0.827 & -8.3 & 0.477 & 0.1 \\
\hline B.2 & 0.792 & -12.3 & 0.496 & 4.1 \\
\hline C.1 & 0.846 & -6.2 & 0.442 & -7.4 \\
\hline C. 2 & 0.877 & -2.8 & 0.406 & -15.4 \\
\hline
\end{tabular}

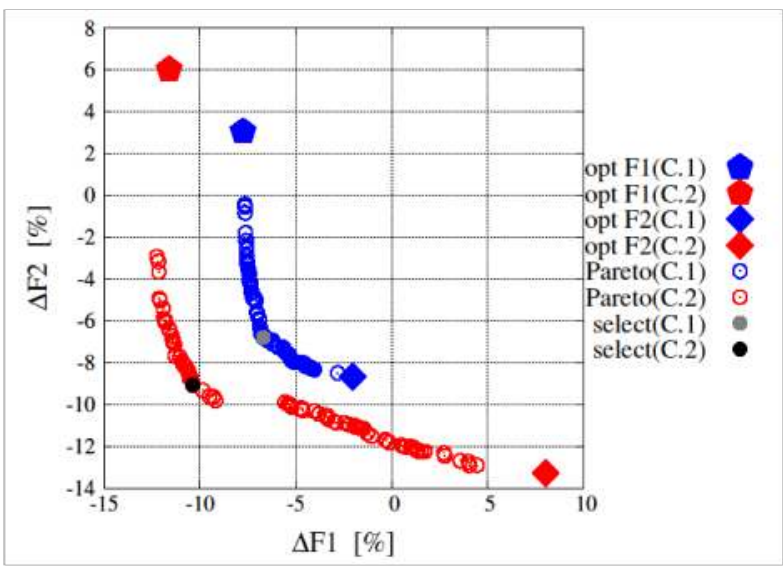

Figure 5. INSEAN/UI - Pareto fronts for design space C.1 and C.2

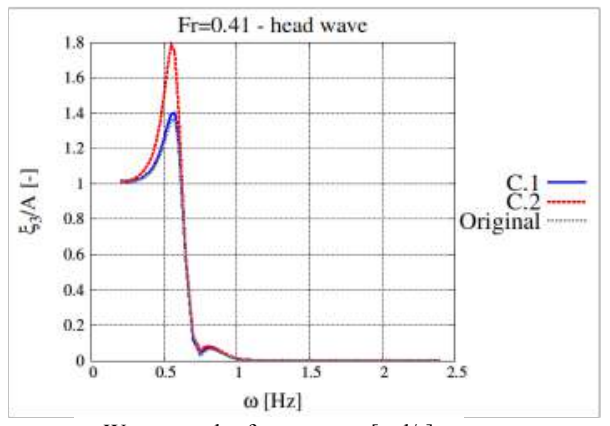

Wave angular frequency $\omega[\mathrm{rad} / \mathrm{s}]$

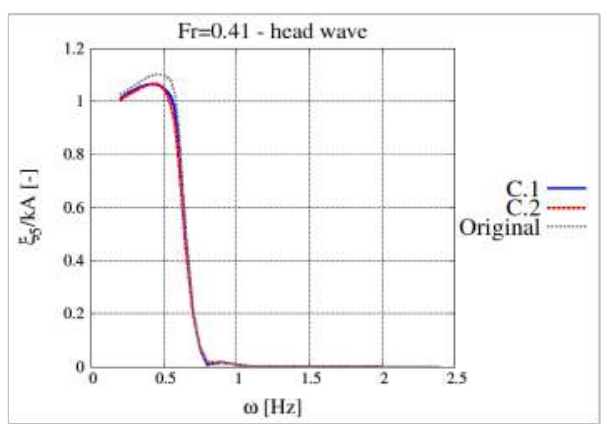

Wave angular frequency $\omega[\mathrm{rad} / \mathrm{s}]$

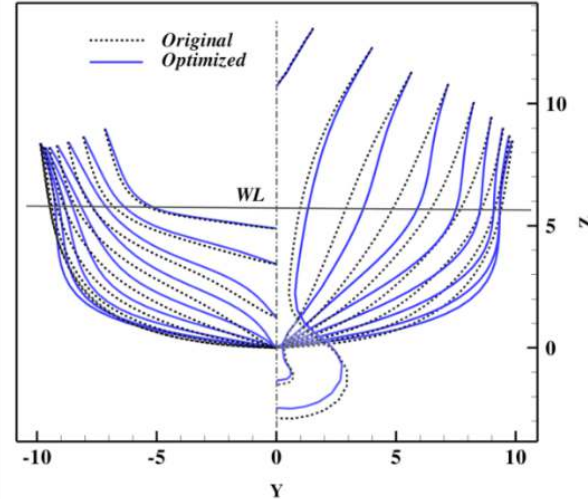

Figure 6. INSEAN/UI - Selected optimal shape for design space C.1

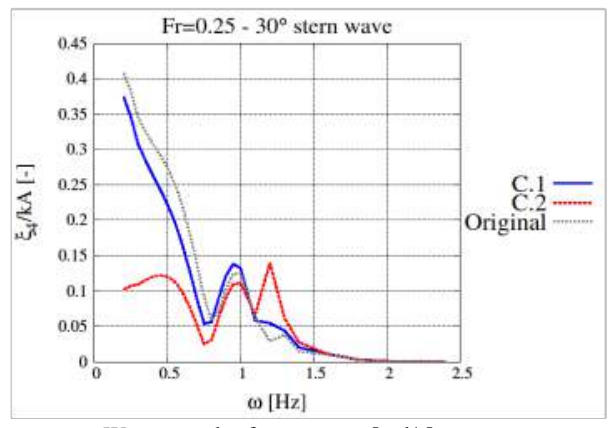

Wave angular frequency $\omega[\mathrm{rad} / \mathrm{s}]$

Figure 7. INSEAN/UI - RAOs of heave (a) and pitch (b) at $\mathrm{Fr}=0.41$ in head wave, and roll (c) at $\mathrm{Fr}=0.25$ in 30 deg stern wave 


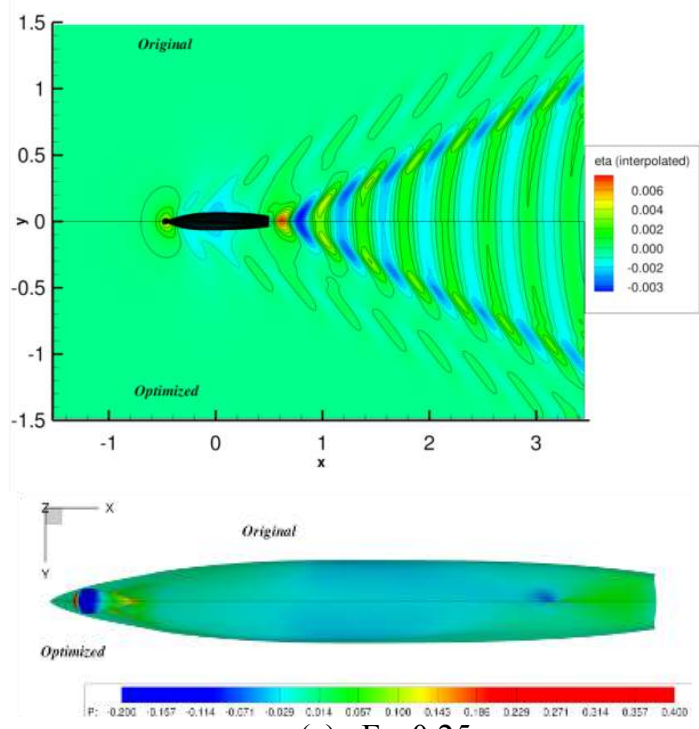

(a) $\mathrm{Fr}=0.25$
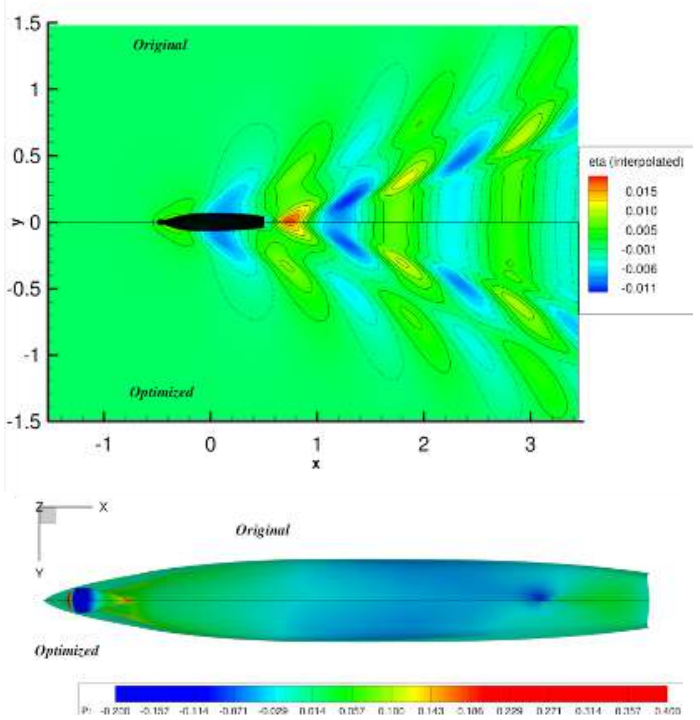

(b) $\mathrm{Fr}=0.41$

Figure 8. INSEAN/UI - Wave elevation pattern and pressure field of the selected optimal hull for design space C.1, compared with the original

a significant penalization in terms of the heave motion response (Fig. 7a), whereas B.2 design provides an RMS of the roll motion worse than the original (Table 6). For these reasons, the C.1 optimal hull is selected for further investigation by RANSE. Figure 5 shows the Pareto front and the selected solution for design space C. The corresponding hull form (C.1) is shown in Fig. 6, and compared to the original. Finally, Figs. $8 \mathrm{a}$ and $8 \mathrm{~b}$ shows a comparison between the original and the selected optimal hull (C.1) in terms of wave elevation pattern and pressure field at $\mathrm{Fr}=0.25$ and $\mathrm{Fr}=0.41$, respectively.

\section{ITU}

ITU uses a relatively simpler approach for obtaining design modifications (experimental space) and then employs an artificial neural network (ANN) as a metamodel of $F 1-F 2$ experimental area, to define the Pareto front and consequently identify the multi-objective solution. The optimization is performed for the $5415 \mathrm{M}$ model (skeg only).

Table 7. ITU - Computed viscous resistance components of the original hull form scaled model $(\mathrm{LBP}=20 \mathrm{~m})$

\begin{tabular}{|c|c|c|c|c|}
\hline Speed $(\mathrm{kn})$ & $\mathrm{Re} \times 10^{7}$ & $R_{f}(\mathrm{~N})$ & $1+k$ & $R_{v}(\mathrm{~N})$ \\
\hline 18 & 6.85 & 762.4 & 1.103 & 841.1 \\
\hline 30 & 11.5 & 1975.2 & 1.109 & 2190.5 \\
\hline
\end{tabular}

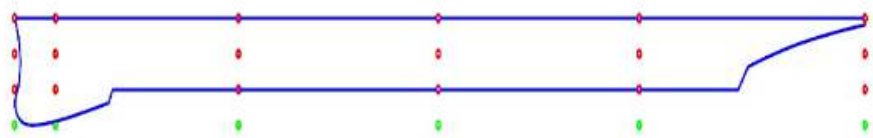

Figure 9. ITU - Design variables (control points) on the hull

\section{ITU - Low-fidelity Solvers}

ITU-Dawson. An in-house potential flow-solver, namely ITUDawson, is used to perform the wave resistance analyses. The unknown velocity potential is calculated by using a source/sink distribution over the panels, distributed on the wetted surface of the ship as well as on a portion of the free-surface in the vicinity of the hull. The free-surface condition is linearized according to the low-Froude number theory. Free-surface condition is satisfied by means of a numerical scheme introduced by Dawson (1977).

Actual (dynamic) sinkage and trim can be applied to the hull by an iterative procedure. Nevertheless, the wave resistance of the variant forms are calculated in fixed condition in the present study. Viscous resistance is approximated as $(1+k) R_{f}$, where the form factor $(1+k)$ is assumed to be fixed as obtained from ITU viscous analysis (see Table 7).

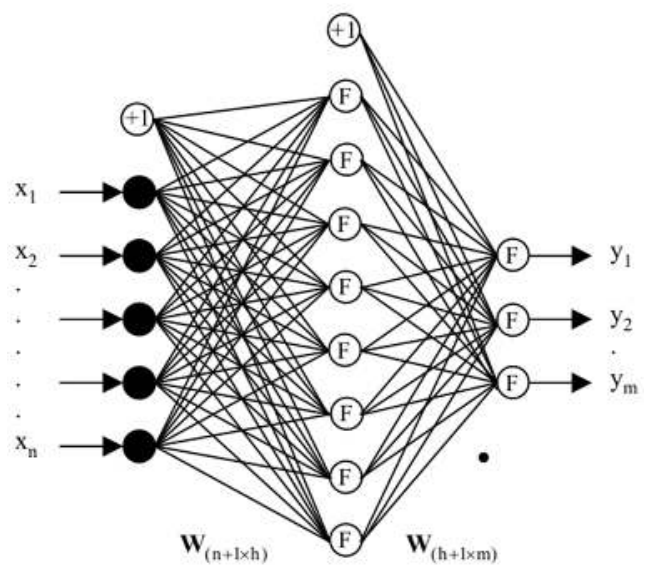

Figure 10. ITU - A graphical representation of static ANN during recall period 


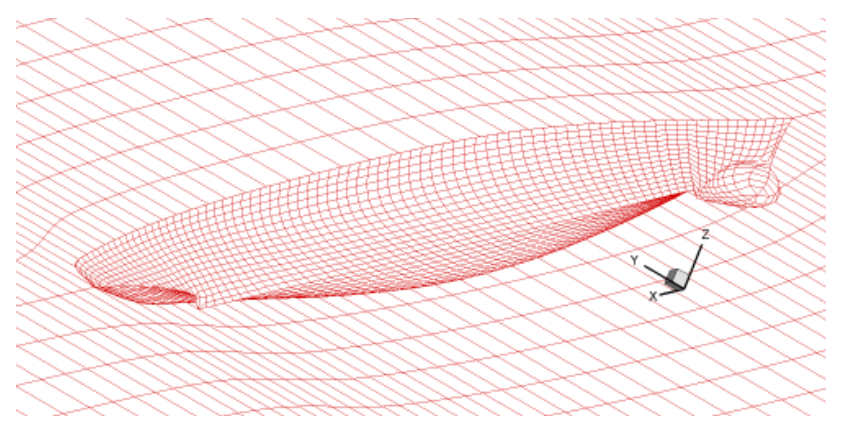

Figure 11. ITU - Panel distribution over the hull and its freesurface vicinity

ITU-SHIPMO. Usual strip theory is employed to obtain vertical acceleration at the bridge at $30 \mathrm{kn}$ in head seas and roll amplitude in $30 \mathrm{deg}$ stern waves at $18 \mathrm{kn}$. The two dimensional added mass and damping coefficients are predicted by using the Frank CloseFit method which is a module in the in-house code ITU-SHIPMO.

\section{ITU - Design Modification Method}

On the one hand, hull-form generation from a parent shape, by variation of basic hull parameters, may not be fruitful because the derived hull forms inherit the characteristics of the parent hull. On the other hand, varying the hull surface points directly may generate fairing issues and generally one has to deal with a very large number of design variables. In order to overcome these difficulties, a simpler approach is adopted, which uses limited number of control points (see Fig. 9) at which randomly distributed relaxation coefficients (between 0.95 and 1.05 in this study) are assigned to modify the hull surface. This means that a $3 \mathrm{D}$ matrix is formed with limited number of rows $\left(\boldsymbol{x}^{(i)}\right)$ and columns $\left(\boldsymbol{z}^{(\boldsymbol{i})}\right)$, and corresponding to this pair of coordinates there is a randomly assigned value of relaxation coefficients $\boldsymbol{C}_{\boldsymbol{r}}$. Akima's (1978) surface generation method, from a set of scattered points, is used to define the surface related to these coefficients; that is, by using $x$ and $z$ coordinates of a particular point:

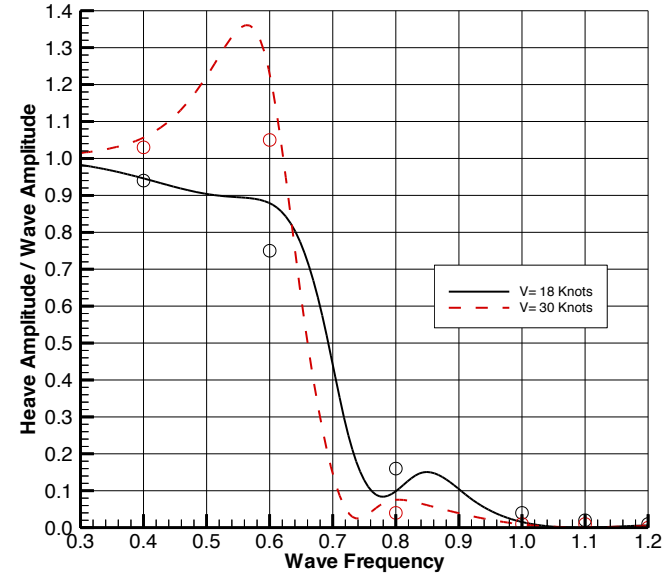

Figure 12. ITU - Predicted and measured (circles) heave RAOs in head seas (180 deg)

$$
y^{(m)}=y^{(i)} \cdot C_{r}\left(x^{(i)}, z^{(i)}\right)
$$

where $\boldsymbol{y}^{(\boldsymbol{m})}$ is the modified value of the initial offset of $\boldsymbol{y}^{(\boldsymbol{i})}$. Furthermore, to have a finer mesh, the relaxation coefficients for other intermediate (interpolation) points are obtained by interpolation using the coefficients defined (assigned) at the control points.

\section{ITU - Optimization Algorithm}

A database of 250 modified hull forms is obtained by means of the design modification method described above. Static artificial neural networks have the capability of storing data during the learning process and then reproducing these data during the recall process. Danisman et al. (2002), Danisman (2014) presented this ANN ability for hull form optimization purposes.

ANN simply establish a functional relationship between $\mathbb{R}^{n}$ and $\mathbb{R}^{m}$, assumed to be input and output data spaces of dimensions $n$ and $m$, respectively. Figure 10 shows the input and output vectors, respectively, as $\boldsymbol{X}=\left(x_{1}, x_{2}, \ldots, x_{n}\right)$ and $\boldsymbol{Y}=$ $\left(y_{1}, y_{2}, \ldots, y_{n}\right)$. The numerical flow solver provides a set of output values, such as wave resistance $\left(R_{w}\right)$, in response to a set of input values (control variables, $x_{1}, x_{2}, \ldots, x_{n}$ ). After a successful training, the ANN can easily and reliably replace the

Table 8. ITU - First 10 hull variants in terms of the regression coefficients for each control point on the hull (from the database used in ANN training process)

\begin{tabular}{|c|c|c|c|c|c|c|c|c|c|c|c|c|}
\hline$x_{1}$ & $x_{2}$ & $x_{3}$ & $x_{4}$ & $x_{5}$ & $x_{6}$ & $x_{7}$ & $x_{8}$ & $x_{9}$ & $x_{10}$ & $x_{11}$ & $x_{12}$ & $F_{\text {Combined }}^{w}$ \\
\hline 1.000 & 1.000 & 1.000 & 1.000 & 1.000 & 1.000 & 1.000 & 1.000 & 1.000 & 1.000 & 1.000 & 1.000 & 1.000 \\
\hline 0.952 & 1.003 & 1.093 & 0.938 & 1.085 & 1.091 & 0.981 & 0.937 & 0.953 & 1.026 & 1.008 & 1.013 & 1.050 \\
\hline 1.003 & 1.074 & 1.001 & 0.988 & 1.016 & 0.983 & 1.048 & 0.951 & 0.922 & 1.097 & 1.071 & 0.986 & 0.952 \\
\hline 1.028 & 0.953 & 1.070 & 0.957 & 0.989 & 0.949 & 0.950 & 0.905 & 0.974 & 0.920 & 0.938 & 0.977 & 1.030 \\
\hline 1.083 & 0.910 & 1.009 & 1.011 & 0.996 & 0.986 & 1.005 & 1.063 & 1.033 & 1.052 & 1.095 & 1.054 & 0.990 \\
\hline 0.942 & 1.053 & 0.952 & 0.927 & 0.919 & 0.910 & 0.933 & 0.920 & 1.084 & 0.977 & 1.001 & 0.949 & 0.908 \\
\hline 1.031 & 1.097 & 1.066 & 1.093 & 0.941 & 0.983 & 0.919 & 1.021 & 0.919 & 1.003 & 1.076 & 0.962 & 1.070 \\
\hline 1.052 & 0.956 & 1.090 & 0.970 & 1.022 & 0.908 & 1.092 & 1.086 & 0.906 & 0.912 & 0.920 & 0.920 & 1.050 \\
\hline 0.956 & 1.035 & 0.999 & 0.920 & 0.993 & 1.085 & 1.040 & 0.990 & 0.961 & 1.001 & 0.903 & 1.064 & 0.935 \\
\hline 0.936 & 0.957 & 0.917 & 1.065 & 1.067 & 1.091 & 1.038 & 1.003 & 1.000 & 1.074 & 1.027 & 0.952 & 0.975 \\
\hline
\end{tabular}



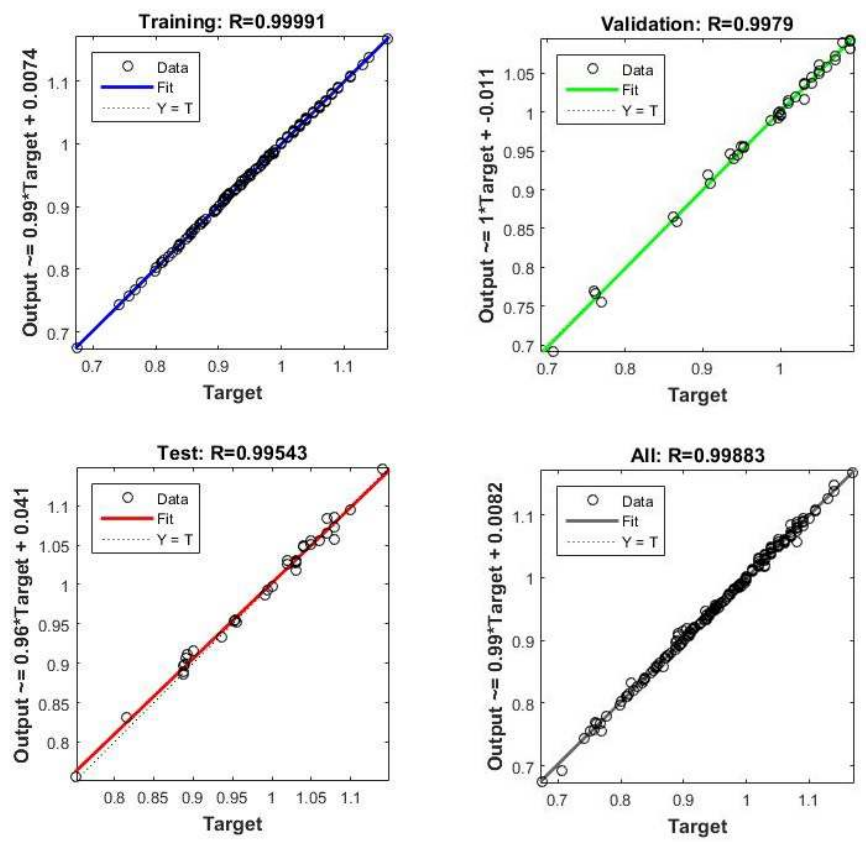

Figure 13. ITU - Regression performance of the ANN training

numerical flow solver. In order to determine the Pareto front out of the experimental area, a simpler and a basic approach is considered where the combined (or aggregate) objective function is expressed as

$$
F_{\text {Combined }}^{w}=w \times F 1+(1-w) \times F 2
$$

where $w=0,0.1, \ldots, 0.9,1.0$ is employed as a weighting factor. The selected optimization algorithm is based on sequential quadratic programming (SQP) within Matlab optimization toolbox, since it is very suitable for constraint optimization problems whose design variables include upper and lower bounds. Schittkowski (1985) showed the success of this algorithm in many aspects such as accuracy, efficiency and

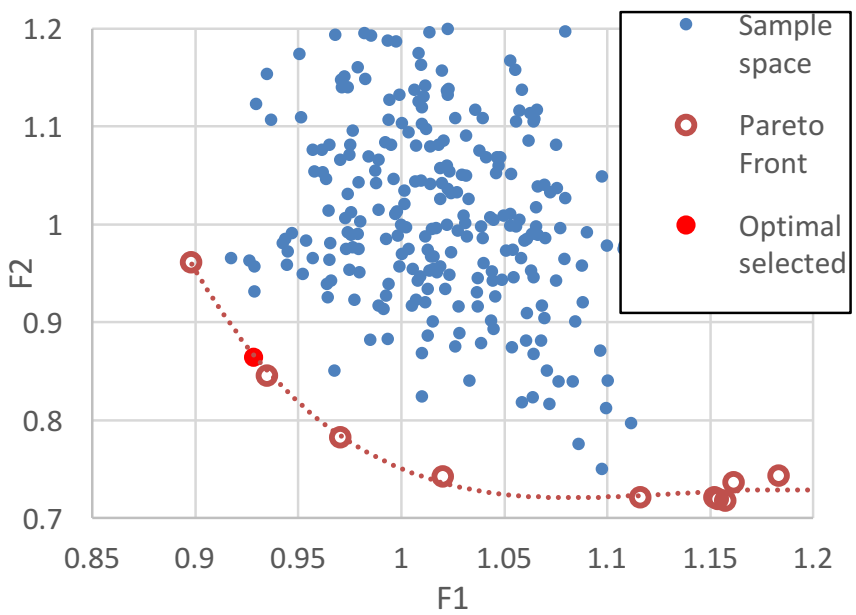

Figure 14. ITU - Experimental area (sample space) and Pareto frontier determined by means of ANN and SQP number of successful solutions over a large number of test problems.

\section{ITU - Numerical Results}

Computational Setup and Results for Resistance and Seakeeping. Grid convergence (or panel-grid sensitivity analysis) is not shown for the present case, since grid studies have been performed on the code ITU-Dawson for similar hull forms. Accordingly, about 1300 panels over the hull surface (demi-hull) and 1600 panels over the free-surface (half symmetric plane) are used. Dimensions of the panelled free-surface are: 1.0 LBP upstream, 1.5 LBP downstream and 0.85 LBP sidewise. Preliminary benchmark tests, with the discretization model given in Fig. 11, show that the code satisfactorily computes wave resistance of the hull forms in consideration. As to the viscous resistance, only the changes in the wetted surface area of the variant hull forms are reflected in the viscous resistance through the frictional resistance, $R_{f}$.

As a representative output of the present code, the predicted and measured heave RAOs for model $5415 \mathrm{M}$ at $18 \mathrm{kn}$ and $30 \mathrm{kn}$ are shown in Fig. 12.

Design Space Definition. Referring to ANN's capability to learn the functional relationship between input and output data sets, a group of 250 randomly selected input values $\left(x_{1}, x_{2}, \ldots, x_{n}\right)$ is used to generate a set of output values. A number of 180 data points are used to train a fully connected ANN with a sigmoid function as its activation function. A number of 35 data points are used in testing and validation during the training process. Remaining 35 data points which are unseen during the training process are evaluated externally by using ANN like an explicit function. To demonstrate the success of the ANN training phase, regression diagrams are given in Fig. 13.

Design Optimization and selection of the optimized hull. Based on 250 variant hull forms and their performances in resistance and seakeeping and on a trained ANN over the sample space, a SQP

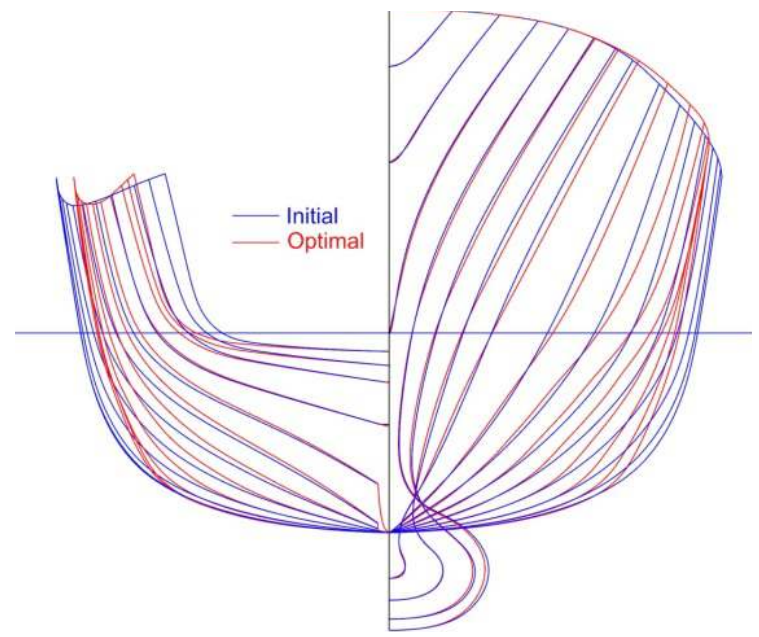

Figure 15. ITU - Comparison of the cross sections of the initial and the optimal hull forms 


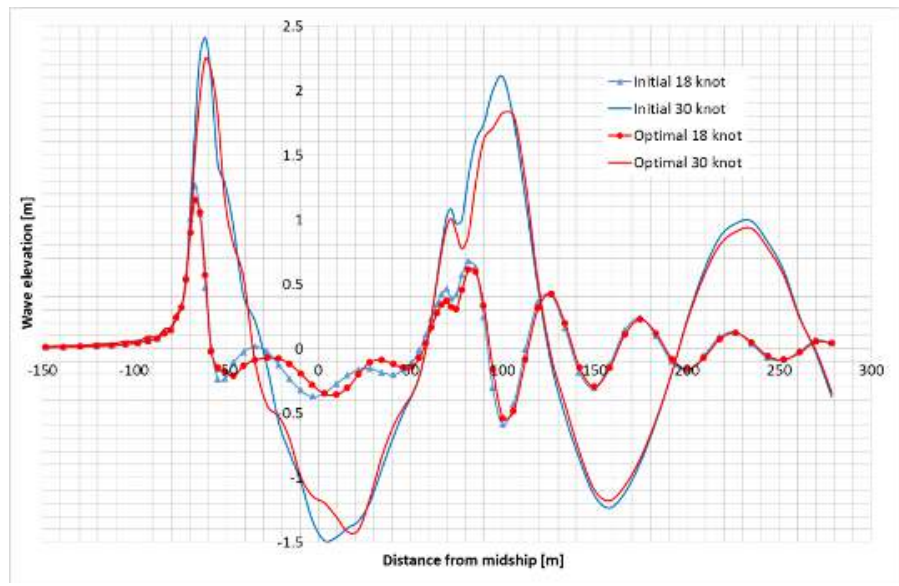

Figure 16. ITU - Wave deformations of the initial and the optimal hull at 18 and $30 \mathrm{kn}$

Table 9. ITU - Comparative seakeeping performances

\begin{tabular}{|l|c|c|}
\hline \multirow{2}{*}{ Hull } & $\begin{array}{c}\mathrm{Fr}=0.41 \\
\text { head wave }\end{array}$ & $\begin{array}{c}\mathrm{Fr}=0.25,30 \mathrm{deg} \\
\text { stern wave }\end{array}$ \\
\cline { 2 - 3 } & $R M S\left(a_{\mathrm{z}}\right)\left(\mathrm{m} / \mathrm{s}^{2}\right)$ & $R M S(\phi)(\mathrm{deg})$ \\
\hline Original & 0.806 & 2.321 \\
\hline Pareto optimum & 0.792 & 2.015 \\
\hline$\Delta \%$ & -1.9 & -13.2 \\
\hline
\end{tabular}

process is carried out to optimize the prescribed aggregate objective function with weighting factors $w$. The optimal forms for each weighting factor $(w)$ are investigated by considering $F_{\text {Combined }}^{w}$ in the ANN training process (see Table 8). The SQP application on the metamodel provided by ANN gives an optimal point, which is expected to be part of the overall Pareto front. An optimal point is selected on the Pareto front according to the changes in the Pareto curve, in order to give favorable results for both $F 1$ and $F 2$. Figure 14 shows $F 1$ vs $F 2$ values of the variant hull forms, the approximated Pareto front and the optimal design. The comparison of the cross sections of the initial and the optimal hull forms can be observed in Fig. 15. Wave deformations of the initial and the optimal hull forms, for 18 and $30 \mathrm{kn}$, are presented in Fig. 16, which points out the success of the selected optimal hull. The contour plot of the wave patterns of the initial and the optimal hull forms can be compared in Fig. 17. The summary of the seakeeping performance of the present multi-objective

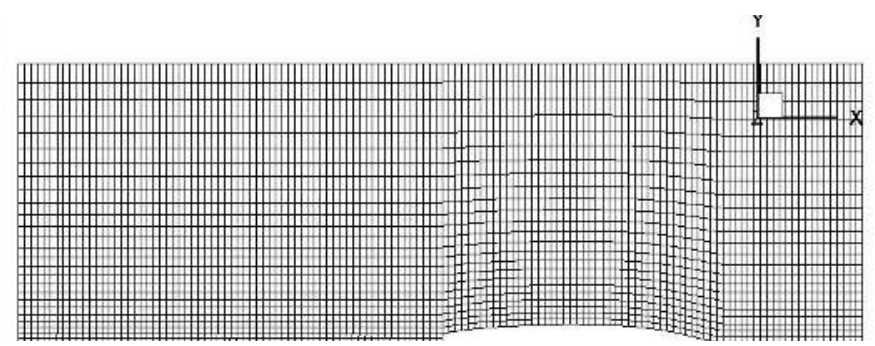

(a) Free-surface

Figure 18. NTUA - Spline sheet on the computational grid

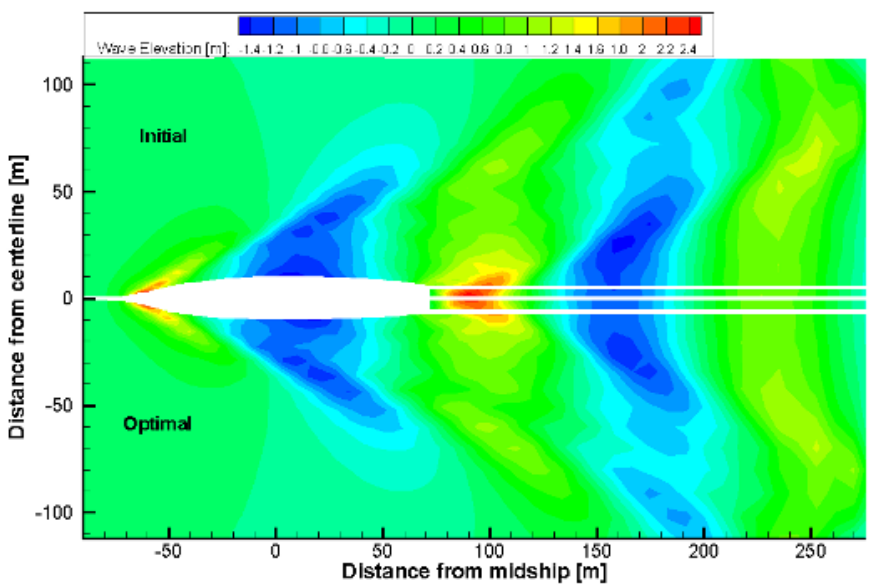

Figure 17. ITU - Contour plot of the wave patterns of the initial and the optimal hull forms $(30 \mathrm{kn})$

Table 10. ITU - Performance of the solution out of the multi-objective problem

\begin{tabular}{|l|c|c|}
\hline Hull & $F 1$ & $F 2$ \\
\hline Original & 1.000 & 1.000 \\
\hline Pareto optimum & 0.928 & 0.865 \\
\hline$\Delta \%$ & -7.2 & -13.5 \\
\hline
\end{tabular}

process is given in Table 9 and the final overall performance in terms of $F 1$ and $F 2$ is summarized in Table 10.

\section{NTUA}

NTUA integrates the parametric modelling and optimization algorithm of the CAESES/FRIENDSHIP-Framework (FFW) with two low-fidelity solvers for calm water and seakeeping performances (Kring and Sclavounos, 1995; Sclavounos, 1996). Optimization results are shown for the $5415 \mathrm{M}$ model (skeg only).

\section{NTUA - Low-fidelity Solvers}

The hydrodynamic performance of the initial hull form and its variants is evaluated via the commercial SWAN2 2002 code for the calm water and the custom-made code SPP- 86 for the rough water. 
Table 11. NTUA - Upper and Lower values of the design variables

\begin{tabular}{|c|c|c|c|c|c|}
\hline No & Design Variable & Units & $\begin{array}{l}\text { Lower } \\
\text { Value }\end{array}$ & $\begin{array}{c}\text { Initial Value } \\
\text { (Parent) }\end{array}$ & $\begin{array}{l}\text { Upper } \\
\text { Value }\end{array}$ \\
\hline 1 & Maximum Beam at Station 0 & $\mathrm{~m}$ & 6.5 & 6.919 & 7.2 \\
\hline 2 & Maximum beam of sonar dome & $\mathrm{m}$ & 2.9 & 3.2 & 3.6 \\
\hline 3 & Angle of Entrance & deg & 185.3 & 190.3 & 195.3 \\
\hline 4 & Wedge Depth & $\mathrm{m}$ & 0.0 & 0.0 & 0.6 \\
\hline 5 & Maximum Beam at WL & $\mathrm{m}$ & 9.0 & 9.528 & 9.8 \\
\hline 6 & Aft longitudinal position of sonar dome's lower profile & $\mathrm{m}$ & 125.8 & 126.2 & 126.5 \\
\hline 7 & Forward longitudinal position of sonar dome's lower profile & $\mathrm{m}$ & 140.8 & 141.9 & 142.5 \\
\hline 8 & Height of Skeg & $\mathrm{m}$ & 1.5 & 1.958 & 2.5 \\
\hline 9 & Longitudinal position of sonar dome's maximum beam & $\mathrm{m}$ & 135.8 & 136.0 & 138.0 \\
\hline 10 & $\begin{array}{l}\text { Sonar dome's tip elevation (for the most forward point of sonar } \\
\text { dome's length) }\end{array}$ & $\mathrm{m}$ & -1.7 & -1.3 & -1.3 \\
\hline
\end{tabular}

$\boldsymbol{S W A N 2}$ 2002. The Ship Wave ANalysis is a software package for the hydrodynamics analysis developed at MIT (Kring and Sclavounos, 1995). SWAN2 2002 distributes quadrilateral panels over the ship hull and the free surface to derive numerically the steady and unsteady free-surface potential flow around ships, using a three-dimensional Rankine Panel Method in the time domain. Only the calm water results are used in the present study. A batch file is used to integrate SWAN2 with the CAESES/FFW. Additional calculations in calm water have been carried out via a potential flow code developed in-house at Laboratory for Ship and Marine Hydrodynamics (LSMH) of NTUA (Tzabiras, 2008). The code solves the non-linear potential flow around the ship, by calculating iteratively the free surface, while both the dynamic and kinematic conditions are satisfied on it. The free surface and the solid boundary are covered by quadrilateral elements. The distribution of panels varies, being denser near geometrical discontinuities and areas of expected high pressures. The Laplace equation is solved according to the classical Hess and Smith (1968) method. An iterative Lagrangian procedure is adopted to cope with the non-linear problem, in conjunction with an Eulerian solution of the vertical momentum equation. Table 12 has been derived on the basis of this method.

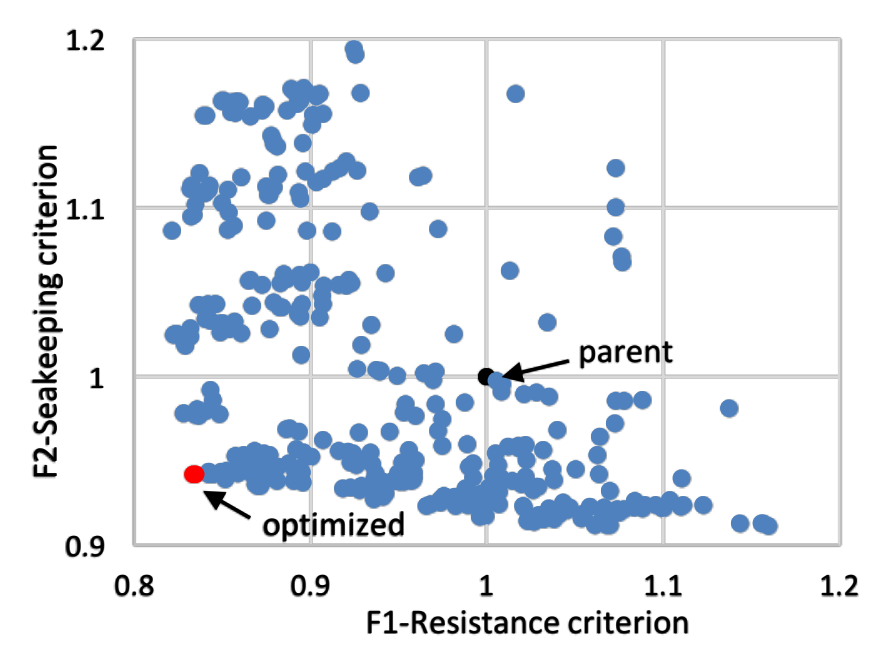

(a)
SPP-86. The seakeeping qualities of the parent and the variant hull forms are calculated by the SPP-86 code, developed at LSMH of NTUA to implement the Salvensen, Tuck and Faltinsen (1970) strip theory. The code distributes Kelvin sources along the wetted part of each ship section following Frank (1967) method. The estimated dynamic responses encompasses vertical and lateral motions, velocities and accelerations and velocities at specific points for a variety of wave frequencies and heading angles.

\section{NTUA - Design Modification Method}

Within CAESES/FFW, the geometry of the hull form is represented by a set of basic curves, providing topological information (design waterline, centerline, deck-line) and a set of 19 section curves. All of them are either F-splines, or B-splines. The hull surface is generated by interpolating the parametricmodelled section curves. The parent hull is split into three regions: the main hull, the sonar dome and the skeg, assigning specific design variables for each of them. A total number of ten design variables is employed, five of which are used to define the sonar dome. Table 11 includes the upper and lower values of the design variables.

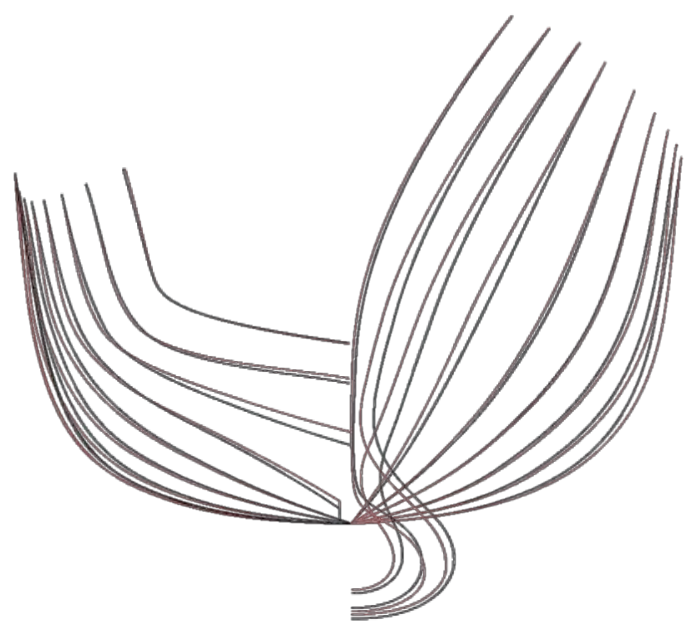

(b)

Figure 19. NTUA - Hull variants of the multi-objective optimization (a), and body plans of the (red) optimized hull compared to the (black) parent hull (b) 
The variation of the hull geometries is made by simply giving an upper and a lower value of the chosen design variables, to the CAESES/FFW environment. The aforementioned boundaries are selected after inspection that they refer to smooth hull shapes. In addition, they comply with the DTMB 5415 geometrical constraints.

\section{NTUA - Optimization Algorithm}

The NSGA II (Non-Dominated Sorting Genetic Algorithm II; Deb, 2002) has been selected for the optimization process. The procedure of this algorithm is described below:

1. A number of variant geometries is generated.

2. An equal number of off-springs is formed.

3. The total number of parents and offspring is sorted to levels according to non-domination.

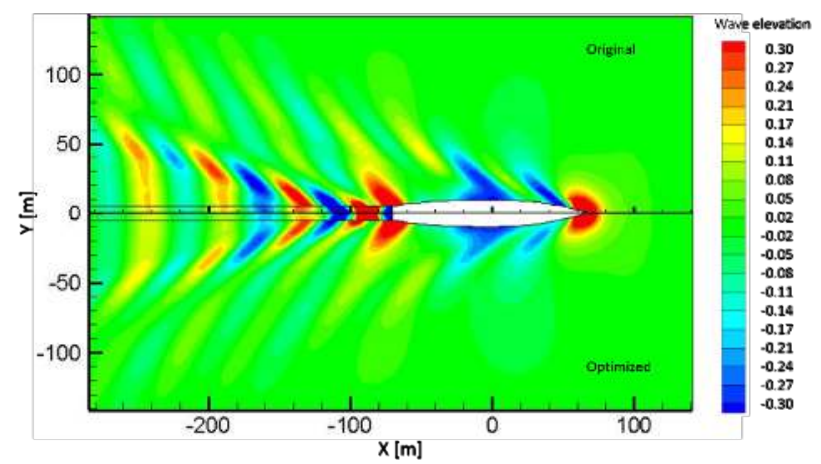

Figure 20. NTUA - Comparison of the wave elevation generated by the initial and the optimized geometry for the $\mathrm{Fr}=0.25$

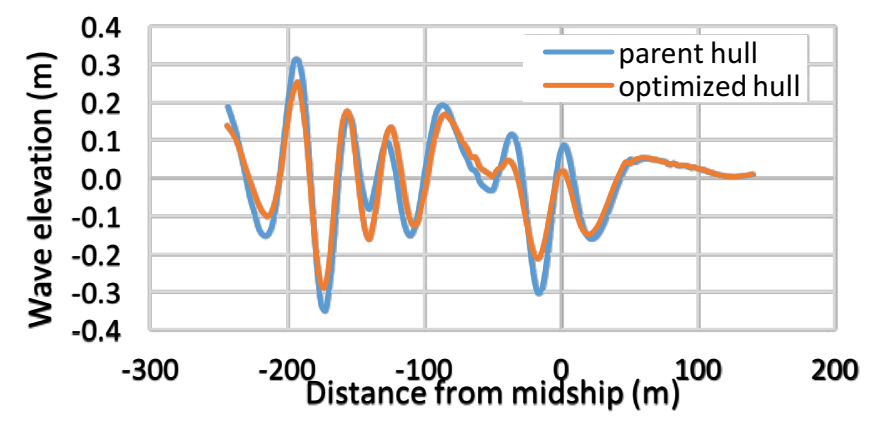

(a) $y / \mathrm{LBP}=0.255$

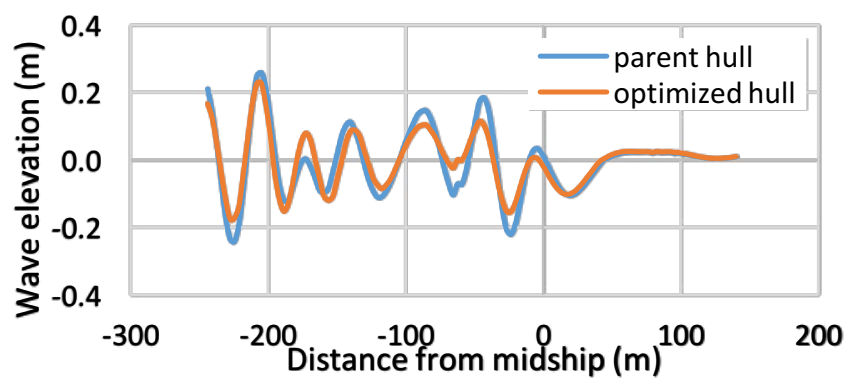

(c) $y / \mathrm{LBP}=0.300$
4. The geometries of each level are ranked with respect to their crowded distance of each solution in the population.

5. A new generation is being produced with a population number equal to the initial one.

6. Steps 2 to 5 are repeated.

The diversity among non-dominated solutions is introduced by using the crowding comparison procedure where for two different solutions $p$ and $q, p$ dominates $(p<q)$ if the following is attained:

$$
\left\{\begin{array}{l}
f_{j}\left(x_{1}\right) \leq f_{j}\left(x_{2}\right), \quad \forall j \in\{1, \ldots, n\} \\
f_{k}\left(x_{1}\right)<f_{k}\left(x_{2}\right), \quad \forall k \in\{1, \ldots, n\}
\end{array}\right.
$$

where $x_{1}$ and $x_{2}$ represent the design variables for $p$ and $q$ geometries respectively.

Table 12. NTUA - Comparative seakeeping performances of the hulls

\begin{tabular}{|l|c|c|}
\hline \multirow{2}{*}{ Hull } & $\begin{array}{c}\text { Fr }=0.41 \\
\text { head wave }\end{array}$ & $\begin{array}{c}\text { Fr }=0.25,30 \mathrm{deg} \\
\text { stern wave }\end{array}$ \\
\cline { 2 - 3 } & $R M S\left(a_{z}\right)\left(\mathrm{m} / \mathrm{s}^{2}\right)$ & $R M S(\phi)(\mathrm{deg})$ \\
\hline Original & 0.982 & 0.874 \\
\hline Pareto optimum & 0.990 & 0.766 \\
\hline$\Delta \%$ & 0.81 & -12.3 \\
\hline
\end{tabular}

Table 13. NTUA - Performance of the solution out of the multi-objective problem

\begin{tabular}{|l|c|c|}
\hline Hull & $F 1$ & $F 2$ \\
\hline Original & 1.000 & 1.000 \\
\hline Pareto optimum & 0.834 & 0.942 \\
\hline$\Delta \%$ & -16.59 & -5.77 \\
\hline
\end{tabular}

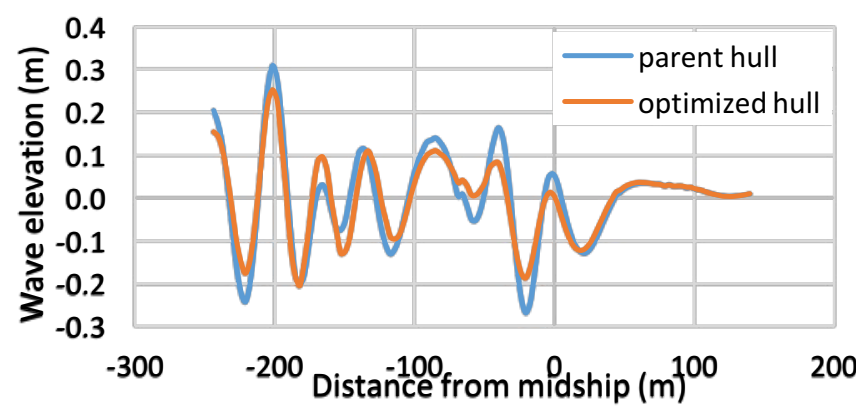

(b) $y / \mathrm{LBP}=0.260$

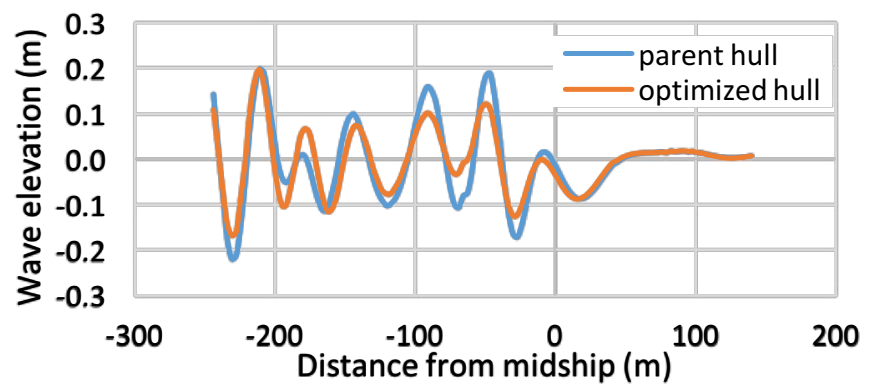

(d) $y / \mathrm{LBP}=0.330$

Figure 21. NTUA - Wave deformations of the parent and optimized hull at $18 \mathrm{kn}$ 
More information is provided by Deb (2002). A number of 25 generations and a population size of 16 are selected, whereas the mutation and the crossover probability are equal to 0.01 and 0.9 , respectively.

\section{NTUA - Numerical Results}

Panel mesh generation. The panel mesh generation of the freesurface and the body surface of the hull is an internal routine of SWAN2 2002. The spline sheet of the body surface is defined by 45 nodes in a direction parallel to the $x$-axis, corresponding to a number of 44 panels in the $x$ direction, and by 13 nodes athwarships. The domain of the free surface is defined by $0.5 \mathrm{LBP}$ upstream, 1.5 LBP downstream and 1 LBP along the transverse distance. Figure 18 presents the spline sheet of the free-surface (a) and the body (b), respectively.

Design Optimization and selection of the optimized hull. The solutions with respect to both the resistance $(F 1)$ and the seakeeping $(F 2)$ criterion, calculated in SWAN2, are shown in Fig. 19a. The solutions concern the variation of 400 hull forms produced by NGSA-II, The selected optimal (red) and the parent hull (black) are also shown in Fig. 19a. Figure 19b depicts a comparison between the body plans of the parent and the optimized hull. Figure 20 shows the comparison of the contour plot of the wave elevation between the initial and the optimized hull at $18 \mathrm{kn}$.

Even thought the $F 1$ objective function concerns the total resistance (including the wave resistance computed by the potential flow code), a comparison between the height of waves generated in specific longitudinal cuts along the ship for the parent and the optimized hull has been made. Figure 21 presents the profile of the waves generated by the initial hull, for $y / \mathrm{LBP}=$ (a) 0.225 , (b) 0.260 , (c) 0.300 , (d) 0.330 .

The origin of the coordinate system is assumed amidships and on the free surface. Table 12 summarized summarized the optimization results for $F 2$ in terms of $R M S$ vertical acceleration at the bridge and roll motion. $F 1$ and $F 2$ objectives improvements are summarized in Table 13.

\section{ECN}

The role of ECN/CNRS is to verify the performances of the optimized hulls by high-fidelity computations.

\section{ECN - High-fidelity Solver}

ISIS-CFD is a flow solver for the incompressible unsteady Reynolds-averaged Navier-Stokes equation (RANSE), available as a part of the FINETM/Marine computing suite. The solver is fully implicit, based on the finite volume method to build the spatial discretization of the transport equations. Surface and volume integrals are evaluated according to second-order accurate approximations and the unstructured discretization is face-based. Time derivatives are evaluated using three-level Euler second-order accurate approximations. While all unknown state variables are cell-centered, the systems of equations used in the implicit time stepping procedure are constructed face by face. Fluxes are computed in a loop over the faces and the contribution of each face is then added to the two cells next to the face. This technique poses no specific requirements on the topology of the cells. Therefore, the grids can be completely unstructured; cells with an arbitrary number of arbitrarily-shaped faces are accepted.

Pressure-velocity coupling is obtained through a Rhie and Chow (1983) SIMPLE type method: in each time step, the velocity updates come from the momentum equations and the pressure is given by the mass conservation law, transformed into a pressure equation. In the case of turbulent flows, transport equations for the variables in the turbulence model are added to the discretization. Free-surface flow is simulated with a multi-phase flow approach: the water surface is captured with a conservation equation for the volume fraction of water, discretized with specific compressive discretization schemes (Queutey and Visonneau, 2007).

Table 14. ECN - Forces and Motions from Full Scale RANSE of the hulls in calm water

\begin{tabular}{|l|c|c|c|c|c|c|c|c|c|r|r|r|}
\hline & \multicolumn{3}{|c|}{$C_{T} \times 10^{-3}$} & \multicolumn{3}{c|}{$\mathrm{S}_{\mathrm{w}, \mathrm{stat}}\left(\mathrm{m}^{2}\right)$} & \multicolumn{3}{c|}{$\delta(\mathrm{m})$} & \multicolumn{3}{c|}{$\tau(\mathrm{deg})$} \\
\hline Fr & Ori. & Opt. & $\Delta \%$ & Ori. & Opt. & \multicolumn{1}{c}{$\Delta \%$} & Ori. & Opt. & \multicolumn{1}{c|}{$\Delta \%$} & Ori. & Opti. & \multicolumn{1}{c|}{$\Delta \%$} \\
\hline 0.25 & 2.738 & 2.683 & -2.03 & 2991 & 2994 & 0.12 & 0.189 & 0.186 & -1.62 & 0.086 & 0.138 & 60.46 \\
\hline 0.41 & 5.025 & 5.556 & 10.55 & 2991 & 2994 & 0.12 & 0.619 & 0.622 & 0.51 & -0.376 & -0.080 & -78.86 \\
\hline
\end{tabular}

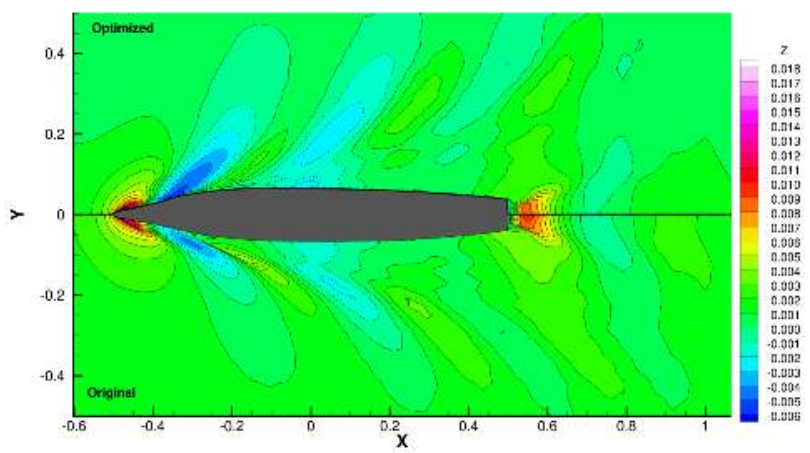

(a) $\mathrm{Fr}=0.25$

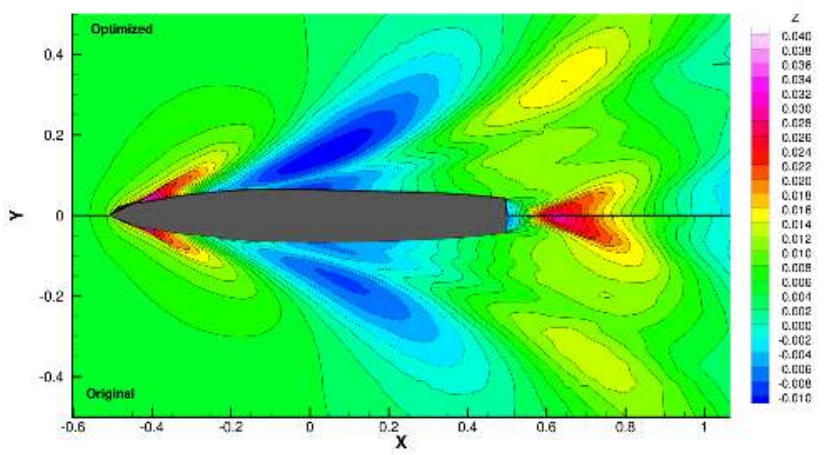

(b) $\mathrm{Fr}=0.41$

Figure 22. ECN - Wave elevations from Full Scale RANSE 
The method features sophisticated turbulence models: apart from classical one equation and two-equation $k-\varepsilon$ and $k-\omega$ models (Menter, 1993), the anisotropic two-equation Explicit Algebraic Reynolds Stress Model (EARSM) (Deng et al., 2006), as well as hybrid RANSE-LES (Large-Eddy Simulation) (Guilmineau et al., 2013). The technique included for the 6 DOF simulation of ship motion is described by Leroyer and Visonneau (2005). Timeintegration of Newton's laws for the ship motion is combined with analytical weighted or elastic analogy grid deformation to adapt the fluid mesh to the moving ship.

Parallelism is based on domain decomposition. The grid is divided into different partitions, these partitions contain the cells. The interface faces on the boundaries between the partitions are shared between the partitions; information on these faces is exchanged with the MPI (Message Passing Interface) protocol. This method works with the sliding grid approach and the different sub-domains can be distributed arbitrarily over the processors.

\section{ECN - Numerical Results}

ECN/CNRS has verified the optimized geometry by INSEAN/UI (Fig. 6). At the current stage, the verification includes only calm water performance of the original and optimized C.1 geometries, in full scale, and at moderate and high Froude numbers $(\mathrm{Fr}=0.25$ and 0.41 ). The hull performances are computed in a free to sink and trim condition by combining the free-surface and moving mesh capabilities with a rigid body motion solver for the flow/motion interaction.
The numerical settings and physical modelling are those of RANSE simulation with a wall function approach with the SST $k-\omega$ turbulence model. The target $y^{+}$value on the walls is 300 , suited for Reynolds number $\mathrm{Re}=1.2 \times 10^{9}$ for $\mathrm{Fr}=0.25$, and $\mathrm{Re}=2 \times 10^{9}$ for $\mathrm{Fr}=0.41$. For symmetry consideration, only $y$ symmetric boat is considered. The mesh is generated with the hexahedral HEXPRESSTM mesh generator with anisotropic grid refinement close to water plane at rest in order to resolve the freesurface deformations for interface capturing method (both air and water solved). A similar grid is used for the two geometries with $1.3 \mathrm{M}$ cells and about $80 \mathrm{~K}$ cells on the bare hull. The vertical resolution of the free-surface is about $15 \mathrm{~cm}$ corresponding to 0.001 LBP. The computation starts with the ship in even keel position and in prescribed draft of $0.0433 \mathrm{LBP}$.

Table 14 summarizes the normalized computed forces and motions to reach the final equilibrium state. Concerning the EFD data for the original geometry in model scale (from IIHR; Longo and Stern, 2005), only the trim angle and the sink can be compared with the CFD values, under the hypothesis that the scale effect is weak on these quantities. EFD predicts a trim angle of $0.085 \mathrm{deg}$ at $\mathrm{Fr}=0.25$ and $-0.421 \mathrm{deg}$ at $\mathrm{Fr}=0.41$ and the measured sink value is 0.189 at $\mathrm{Fr}=0.25$ and 0.619 at $\mathrm{Fr}=0.41$. Under the aforementioned hypothesis, this is in agreement with the CFD values from Table 14. It may be noted that the computed trim is close to the experiments for both RANSE and potential flow; for the computed sinkage, the agreement appears correct with RANSE, whereas it is under predicted at both speeds with the potential flow (see Table 3 ).
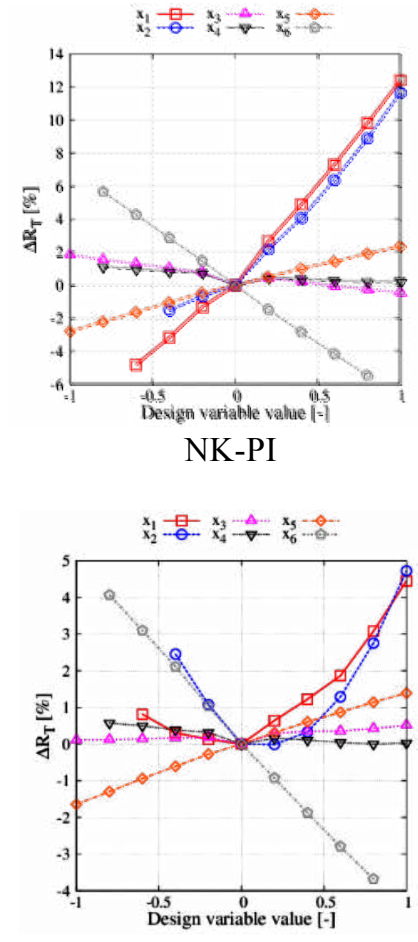

NK-PI

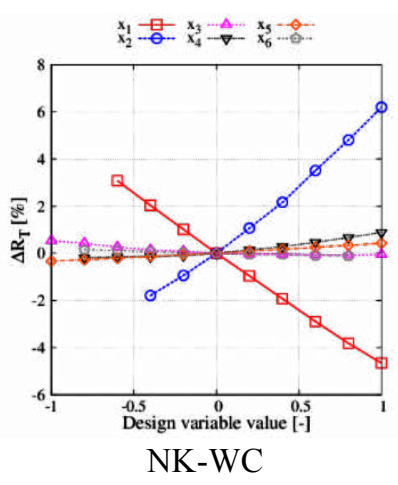

(a) $\mathrm{Fr}=0.25$

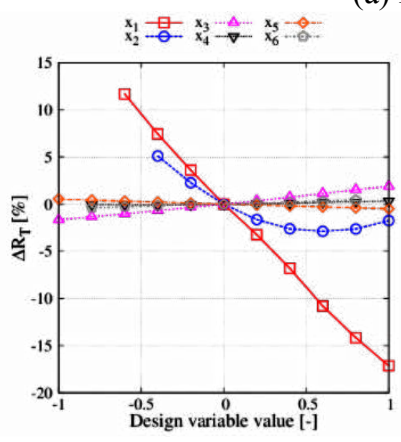

NK-WC

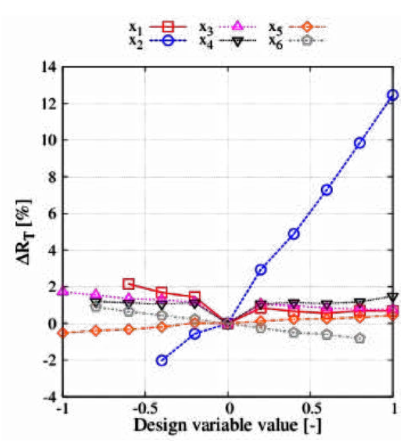

DM-PI

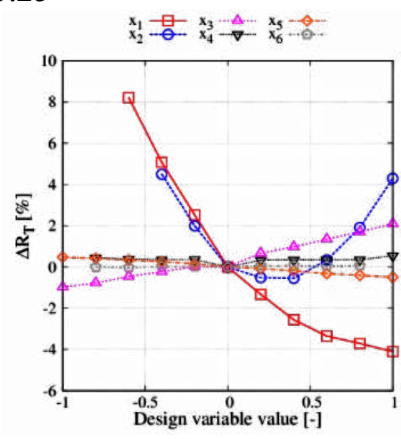

DM-PI

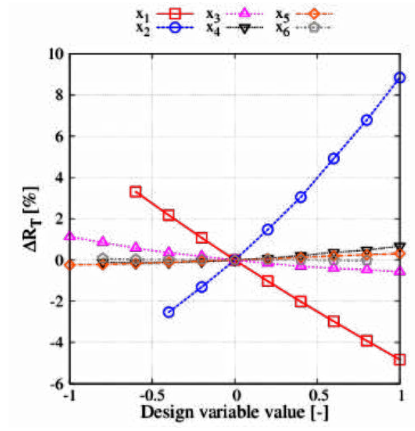

DM-WC

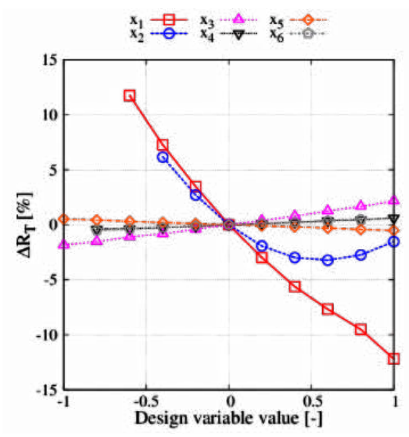

DM-WC

(b) $\mathrm{Fr}=0.41$

Figure 23. INSEAN/UI - Effects of the low-fidelity solver on the optimization outcomes: preliminary sensitivity analysis for total resistance improvement in calm water 
Concerning the forces, it results that with RANSE the resistance coefficient is decreased by $2 \%$ at $\mathrm{Fr}=0.25$, and is increased by $10 \%$ at $\mathrm{Fr}=0.41$. The improvement for $F 1$, as calculated by RANSE, reduces significantly, providing a final weighted resistance reduction by $0.2 \%$. Figure 22 shows a comparison of the wave elevation patterns for the original and the optimized geometry at $\mathrm{Fr}=0.25$ (a) and $\mathrm{Fr}=0.41$ (b), respectively. At the lower Froude number, the bow waves are similar although less pronounced with the optimized geometry. Two other effects can explain the predicted reduced resistance of the optimized hull: a more pronounced wave through close to the bow and a reduced level of the stern wave. At $\mathrm{Fr}=0.41$ a significant breaking bow wave is detected on the optimized hull with an increased wave through at mid-ship.

\section{CONCLUSIONS AND FUTURE WORK}

The paper presented a multi-objective hull form optimization of the DTMB 5415 (specifically the MARIN variant 5415M, with skeg only), performed by three different research team (INSEAN/UI, ITU, and NTUA) within the NATO RTO Task Group AVT-204 to the aim of "Assess the Ability to Optimize Hull Forms of Sea Vehicles for Best Performance in a Sea Environment." Low-fidelity solvers, such as potential flow/strip theory methods, have been used to assess and improve the calm water $(F 1)$ and the seakeeping $(F 2)$ performances at two speeds $(\mathrm{Fr}=0.25$ and $\mathrm{Fr}=0.41)$ and two heading (head and stern wave) at sea state 5 , respectively. The results have been partially verified by ECN/CNRS by high-fidelity simulations using a RANSE solver.

Overall, optimization achievements by low-fidelity solvers have been found significant, with an average improvement for calmwater resistance and seakeeping performances of 10 and $9 \%$ respectively. Moreover, the most promising designs have shown up to $16 \%$ improvement for the calm-water resistance and $14 \%$ for the seakeeping merit factor. The design-space size ranged from two to twelve and the optimized designs show a quite large variability and different characteristic (Figs. 6, 15, and 19), which will be investigated and compared in detail by high-fidelity solvers in future work.

INSEAN/UI has defined six design spaces with dimensionality ranging from two to six, using a linear expansion of orthogonal basis functions for the modification of the DTMB 5415 bare hull. The optimization is performed by a multi-objective extension of the deterministic particle swarm optimization algorithm. The most promising design is identified, showing an improvement of nearly $6.7 \%$ and $6.8 \%$ for $F 1$ and $F 2$ respectively.

ITU has produced 250 hull form variants of the $5415 \mathrm{M}$ using Akima's surface generation, with randomly distributed relaxation coefficients at control points over the body surface. The optimization procedure combines an artificial neural network with a sequential quadratic programming algorithm, which is fed with aggregate objective functions. The selected optimal hull has achieved an improvement of $7.2 \%$ and $13.5 \%$ for $F 1$ and $F 2$, respectively.

NTUA has used the parametric modelling of the CAESES/FRIENDSHIP-Framework for the design modification of the $5415 \mathrm{M}$, representing the hull form by a set of basic curves, providing topological information, and defining a set of 19 sections. The hull surface is parametrized by ten design variables. The NSGA II code is used for the optimization procedure. The selected optimal hull has reached an improvement of nearly $23.4 \%$ and $5.8 \%$ for $F 1$ and $F 2$, respectively.

ECN/CNRS has verified the parent and the INSEAN/UI optimal hull for the calm water performances, using an in-house highfidelity solver (ISIS-CFD). The CFD results have shown a resistance reduction of $2 \%$ at $\mathrm{Fr}=0.25$ and an increment of $10 \%$ at $\mathrm{Fr}=0.41$, proving an overall $0.2 \%$ reduction for $F 1$. Comparing to ISIS-CFD, the calm water resistance evaluated by the potential flow code WARP shows a -3.6 and $-7.1 \%$ error for the original and optimized hull respectively, at $\mathrm{Fr}=0.25$; the error at $\mathrm{Fr}=0.41$ is 21 and $-5.8 \%$ for original and optimized hull, respectively. This has motivated further studies on the impact of the low-fidelity solver on the design optimization outcomes.

Further investigations on the effects of potential flow formulation/linearization on the multi-objective optimization of the DTMB 5145 are in progress by INSEAN/UI. A preliminary sensitivity analysis of the design variables for the calm water resistance at $\mathrm{Fr}=0.25$ and 0.41 is shown in Fig. 23, where Neumann-Kelvin (NK) and double model or Dawson (DM) linearization are combined with a standard pressure integral (PI) and the transversal wave cut (WC) method for the wave resistance evaluation. The trend of some of the variables, such as $x_{1}$, is very sensitive to the formulation used, which may lead to inaccurate design optimization solutions. The identification of the proper trend of the design variables remains a critical issue for low fidelity solvers, especially when large shape modifications are produced. This suggests the use of high-fidelity solvers combined with metamodels, in order to increase the accuracy of the design optimization while keeping the computational cost affordable.

Future work includes RANSE simulations by ECN/CNRS of ITU and NTUA optimal designs, as well as the assessment of the maneuvering performance (steady turn) for parent and most promising optimized hulls. In addition, a high-fidelity RANSE SBDO, based on sequential multi-criterion adaptive sampling and dynamic radial basis function (Diez et al., 2015) will be performed by INSEAN/UI. TUHH will address optimal propulsion studies for selected hull forms. The final assessment of the results will be used to draw the final conclusions for the current optimization exercise, and provide recommendations for effective ship optimization procedures.

Beyond the scopes of the current activities, the design optimization of the DTMB 5415 will be extended to stochastic environment and operations by UI and INSEAN, for reduced resistance/power in wave and increased ship operability. The ship performances will be addressed by uncertainty quantification methods for stochastic sea state, speed, and heading and will be optimized by metamodels and multi-objective particle swarm. The results will be compared to the current studies, which represent the deterministic baseline for the future stochastic optimization of the DTMB 5415. 


\section{ACKNOWLEDGMENTS}

The work has been performed in collaboration with NATO RTO Task Group AVT-204 "Assess the Ability to Optimize Hull Forms of Sea Vehicles for Best Performance in a Sea Environment."

CNR-INSEAN and University of Iowa teams are grateful to Dr Woei-Min Lin and Dr Ki-Han Kim of the US Navy Office of Naval Research, for their support through NICOP grant N6290911-1-7011 and grant N00014-14-1-0195. INSEAN team is also grateful to the Italian Flagship Project RITMARE, coordinated by the Italian National Research Council and funded by the Italian Ministry of Education.

ECN/CNRS team gratefully acknowledge GENCI (Grand Equipement National de Calcul Intensif) for the HPC resources under Grant No 2014-21308.

\section{REFERENCES}

1. Akima H., "A Method of Bivariate Interpolation and Smooth Surface Fitting for Irregularly Distributed Data Points." ACM Transactions on Mathematical Software 4 (1978): 148159.

2. Bassanini P., Bulgarelli U., Campana E.F., Lalli F., "The wave resistance problem in a boundary integral formulation." Surv. Math. Ind. 4 (1994): 151-194.

3. Chen X., Diez M., Kandasamy M., Zhang Z., Campana E.F., Stern F., "High-fidelity global optimization of shape design by dimensionality reduction, metamodels and deterministic particle swarm." Engineering Optimization 47(4) (2015):473-494.

4. Danisman D.B., Mesbahi E., Atlar M., Goren O., "A New Hull Form Optimisation Technique For Minimum Wave Resistance." 10th IMAM Congress, Crete, Greece, (13-17 May 2002).

5. Danisman D.B., "Reduction of demi-hull wave interference resistance in fast displacement catamarans utilizing an optimized centerbulb concept." Ocean Englneering 91 (2014):227-234.

6. Dawson C.W., "A Practical Computer Method For Solving Ship-Wave Problems." 2nd Int. Conf. Numerical Ship Hydrodynamics, Berkeley (1977): 30-38.

7. Deb K., "A fast and elitist multiobjective genetic algorithm: NSGA-II." Evolutionary Computation, IEEE Transactions 6(2) (2002): 182-197.

8. Deng G., Queutey P., Visonneau M., "Three-dimensional flow computation with Reynolds stress and algebraic stress models." in Rodi W., Mulas M. eds, Engineering Turbulence Modelling and Experiments 6 (2006): 389-398.

9. Diez M., Peri D., "Robust optimization for ship conceptual design." Ocean Engineering 37(11) (2010): 966-977.

10. Diez M., Volpi S., Serani A., Stern F., Campana E.F., "Simulation-based Design Optimization by Sequential Multi-criterion Adaptive Sampling and Dynamic Radial Basis Functions." In Proc. EUROGEN 2015 - International Conference on Evolutionary and Deterministic Methods for Design, Optimization and Control with Applications to Industrial and Societal Problems, University of Strathclyde, Glasgow, UK, (14-16 Sept. 2015).
11. Frank W., "Oscillation of Cylinders in or Below the Free Surface of Deep Fluids." DTNSRDC, Rep. No. 2375, Washington, D.C. (1967).

12. Hess J.L., Smith A.M.O., "Calculation of potential flow about arbitrary bodies," Prog. Aeraunaut. Sci. 8 (1968): 1136.

13. Guilmineau E., Chikhaoui O., Deng G.B., Visonneau M., "Cross wind effects on a simplified car model by a DES approach.” Computers and Fluids 78 (2013): 29-40.

14. Kandasamy M., Wu P.C., Zalek S., Karr D., Bartlett S., Nguyen L., Stern, F., "CFD based hydrodynamic optimization and structural analysis of the hybrid ship hull," In Proc. SNAME Maritime Convention, Houston, TX, USA (20-25 October 2014).

15. Kring D., Sclavounos P., "Numerical Stability Analysis for Time-Domain Ship Motion Simulations." Journal of Ship Research 39(4) (1995): 313-320.

16. Leroyer A., Visonneau M., "Numerical methods for RANS simulations of a self-propelled fish-like body." Journal of Fluid and Structures 20(3) (2005): 975-991.

17. Longo J., Stern F., "Uncertainty Assessment for Towing Tank Tests With Example for Surface Combatant DTMB Model 5415." J. Ship Research. 49(1) (2005): 55-68.

18. Menter F.R., "Zonal Two-Equations k- $\omega$ Turbulence Models for Aerodynamic Flow." AIAA 93-2906 (1993).

19. Meyers W.G., Baitis A.E., "SMP84: improvements to capability and prediction accuracy of the standard ship motion program SMP81." DTNSRDC/SPD-0936-04 (Sept. 1985).

20. Olivieri A., Pistani F., Avanzini A., Stern F., Penna R., "Towing tank, sinkage and trim, boundary layer, wake, and free surface flow around a naval combatant INSEAN 2340 model." Tech. rep., DTIC Document (2001)

21. Pellegrini R., Campana E.F., Diez M., Serani A., Rinaldi F., Fasano G., Iemma U., Liuzzi G., Lucidi S., Stern F., "Application of derivative-free multi-objective algorithms to reliability-based robust design optimization of a high-speed catamaran in real ocean environment." In Proc. EngOpt2014 - 4th international conference on engineering optimization Lisbon (8-11 Sept. 2014).

22. Queutey P., Visonneau M., "An interface capturing method for free-surface hydrodynamic flows." Computers and Fluids 36(9) (2007): 1481-1510.

23. Rhie C.M., Chow W.L., "Numerical study of the turbulent flow past an airfoil with trailing edge separation." AIAA Journal 21(11) (1983): 1525-1532.

24. Salvesen N., Tuck E.O., Faltinsen O., "Ship Motions and Sea Loads" Trans. SNAME 78 (1970): 250-287.

25. Schlichting H., Gersten K., Boundary-Layer Theory, Springer-Verlag, Berlin (2000).

26. Serani A., Diez M., Leotardi C., Peri D., Fasano G., Iemma U., Campana E.F., "On the use of synchronous and asynchronous single-objective deterministic particle swarm optimization in ship design problems." In Proc. OPT-i 2014 - 1st International Conference in Engineering and Applied Sciences Optimization, Kos, Greece (4-6 June 2014).

27. Serani A., Diez M., Campana E.F., "Single- and multiojective design optimization study for DTMB 5415, based on low-fidelity solvers." INSEAN Tech, rep. 2015-TR-002 (2015a). 
28. Serani A., Fasano G., Liuzzi G., Lucidi G., Iemma U., Campana E.F., Diez M., "Derivative-free global desgin optimization in ship hydrodynamics by local hybridization." In Proc. 14th International Conference on Computer Application and Information Technology in the Maritime Industries, Ulrichshusen, Germany (11-13 May 2015b): 331342.

29. Schittkowski K., "Nlqpl: A Fortran-Subroutine Solving Constrained Nonlinear Programming Problems." Annals Of Operations Research 5 (1985): 485-500.

30. Sclavounos P.D., "Computation of Wave Ship Interactions." Advances in Marine Hydrodynamics, Qhkusu M. ed., Computational Mechanics Publ, (1996).

31. SPP-86. User's Manual, Report No: NAL-114-F-94, Lab. Ships and Marine Hydrodynamics, National Technical Univ. of Athens (1994).

32. SWAN2. User Manual: Ship Flow Simulation in Calm Water and in Waves, Boston Marine Consulting Inc., Boston MA 02116, USA (2002).

33. Tahara Y., Peri D, Campana E.F., Stern F., "Computational fluid dynamics-based multiobjective optimization of a surface combatant using global optimization method," $J$. Marine Science and Technology 13 (2008): 95-116.

34. Telste J.G., Reed A.M., "Calculation of Transom Stern Flows." In Proc. $6^{\text {th }}$ International Conference on Numerical Ship Hydrodynamics (1994): 78-92.

35. Trelea I.C., "The particle swarm optimization algorithm: convergence analysis and parameter selection." Information Processing Letters 85 (2003): 317-325.

36. Tzabiras G., "A method for predicting the influence of an additive bulb on ship resistance," Proceedings of the $8^{\text {th }}$ International Conference on Hydrodynamics, Nantes (2008): 53-60.

37. Wong T.T., Luk W.S., Heng P.A., "Sampling with Hammersley and Halton Points." Journal of Graphics Tools (1997): 9-24. 